\title{
Melatonin improves the germination rate of cotton seeds under drought stress by opening pores in the seed coat
}

\author{
Yandan Bai ${ }^{1}$, Shuang Xiao ${ }^{1}$, Zichen Zhang ${ }^{1}$, Yongjiang Zhang ${ }^{1}$, Hongchun Sun ${ }^{1}$, Ke Zhang ${ }^{1}$, Xiaodan Wang ${ }^{2}$, \\ Zhiying Bai ${ }^{3}$, Cundong Li ${ }^{1}$, Liantao Liu ${ }^{\text {Corresp. } 1}$ \\ ${ }^{1}$ College of Agronomy, HeBei Agricultural University/ State Key Laboratory of North China Crop Improvement and Regulation/ Key Laboratory of Crop \\ Growth Regulation of Hebei Province, Baoding, Hebei Province, China, Baoding, China \\ 2 College of Traditional Chinese Veterinary Medicine, Hebei Agricultrual University, Baoding, China \\ 3 College of Life Science, Hebei Agricultrual University, Baoding, China \\ Corresponding Author: Liantao Liu \\ Email address: liult@hebau.edu.cn
}

The germination of cotton (Gossypium hirsutum L.) seeds is affected by drought stress; however, little is known about the physiological mechanism affecting germination and the effect of melatonin (MT) on cotton seed germination under drought stress. Therefore, we studied the effects of exogenous MT on the antioxidant capacity and epidermal microstructure of cotton under drought stress. The results demonstrated a retarded water absorption capacity of testa under drought stress, significantly inhibiting germination and growth in cotton seeds. Drought stress led to the accumulation of reactive oxygen species (ROS), malondialdehyde (MDA), and osmoregulatory substances (e.g., proline, soluble protein, and soluble sugars); it also decreased the activity of antioxidant enzymes and $\alpha$ amylase. Drought stress inhibited gibberellin acid (GA) synthesis and increased abscisic acid (ABA) content, seriously affecting seed germination. However, seeds pre-soaked with MT $(100 \mu \mathrm{M})$ showed a positive regulation in the number and opening of stomata in cotton testa. The exogenous application of MT increased the germination rate, germination potential, radical length, and fresh weight, as well as the activities of superoxide dismutase (SOD), peroxidase (POD), and $\alpha$-amylase. In addition, MT application increased the contents of organic osmotic substances by decreasing the hydrogen peroxide $\left(\mathrm{H}_{2} \mathrm{O}_{2}\right)$,

superoxide anion $\left(\mathrm{O}_{2}{ }^{-}\right)$, and MDA levels under drought stress. Further analysis demonstrated that seeds pre-soaked with MT alleviated drought stress by affecting the $A B A$ and GA contents. Our findings show that MT plays a positive role in protecting cotton seeds from drought stress. 


\section{Melatonin improves the germination rate of cotton}

\section{2 seeds under drought stress by opening pores in the}

\section{3 seed coat}

4 Yandan Bai ${ }^{1}$, Shuang Xiao ${ }^{1}$, Zichen Zhang ${ }^{1}$, Yongjiang Zhang ${ }^{1}$, Hongchun Sun ${ }^{1}$, Ke Zhang ${ }^{1}$,

5 Xiaodan Wang ${ }^{2}$, Zhiying Bai ${ }^{3}$, Cundong $\mathrm{Li}^{1}$, Liantao $\mathrm{Liu}^{1 *}$

$6{ }^{1}$ College of Agronomy, HeBei Agricultural University/ State Key Laboratory of North China

7 Crop Improvement and Regulation/ Key Laboratory of Crop Growth Regulation of Hebei

8 Province, Baoding, Hebei Province, China

$9{ }^{2}$ College of Traditional Chinese Veterinary Medicine, Hebei Agricultural University, Baoding, 10 Hebei Province, China

$11{ }^{3}$ College of Life Science, Hebei Agricultural University, Baoding, Hebei Province, China 12

13 Corresponding Author:

14 Liantao Liu

15 NO. 289 Lingyusi Street, Baoding, Hebei Province, 071000, China

16 Email address: liultday@126.com

\section{ABSTRACT}

The germination of cotton (Gossypium hirsutum L.) seeds is affected by drought stress; however, little is known about the physiological mechanism affecting germination and the effect of melatonin (MT) on cotton seed germination under drought stress. Therefore, we studied the effects of exogenous MT on the antioxidant capacity and epidermal microstructure of cotton under drought stress. The results demonstrated a retarded water absorption capacity of testa under drought stress, significantly inhibiting germination and growth in cotton seeds. Drought stress led to the accumulation of reactive oxygen species (ROS), malondialdehyde (MDA), and osmoregulatory substances (e.g., proline, soluble protein, and soluble sugars); it also decreased the activity of antioxidant enzymes and $\alpha$-amylase. Drought stress inhibited gibberellin acid (GA) synthesis and increased abscisic acid (ABA) content, seriously affecting seed germination. However, seeds pre-soaked with MT $(100 \mu \mathrm{M})$ showed a positive regulation in the number and opening of stomata in cotton testa. The exogenous application of MT increased the germination 
31 rate, germination potential, radical length, and fresh weight, as well as the activities of 32 superoxide dismutase (SOD), peroxidase (POD), and $\alpha$-amylase. In addition, MT application 33 increased the contents of organic osmotic substances by decreasing the hydrogen peroxide

$34\left(\mathrm{H}_{2} \mathrm{O}_{2}\right)$, superoxide anion $\left(\mathrm{O}_{2}^{-}\right)$, and MDA levels under drought stress. Further analysis 35 demonstrated that seeds pre-soaked with MT alleviated drought stress by affecting the ABA and 36 GA contents. Our findings show that MT plays a positive role in protecting cotton seeds from 37 drought stress.

38 Subjects Agricultural Science; Plant Science

39 Keywords Cotton, Melatonin, Seed germination, Drought stress, Testa, Physiology 40

\section{INTRODUCTION}

42 Drought is a global challenge because of climate warming and increasingly constrained water 43 resources (Jury \& Vaux, 2005). Drought is also one of the main abiotic stress factors limiting the 44 growth and yield of plants (Farooq et al., 2009a), and the changing global climate is making this situation more extreme (Farooq et al., 2013). The response of plants to drought stress are interconnected (Huang et al., 2019); specifically, drought influences many morphological, physiological, and biochemical processes such as stomatal closure, cellular dehydration, the peroxidation of membrane lipids, and reduced antioxidant capacity, affecting plant survival and development (Ahammed et al., 2019a; Huang et al., 2019; Gao et al., 2018). However, plants have evolved complex stress-resistant mechanisms to counteract damage due to drought, which may allow plants to complete their life cycles (Ahammed et al., 2019a).

Germination is a complex process regulated by a series of physical and metabolic events in the life cycle of plants (Leónie \& Maarten, 2008; Xiao et al., 2019). As the initial stage of plant life cycles and the critical stage of seedling establishment, seed germination is extremely sensitive to drought stress (Ibrahim \& Ehab, 2016). A previous study has shown that the mechanical barrier provided by the seed coat increases under stress conditions, indicating that drought stress inhibits seed germination (Debeaujon, Léon-Kloosterziel \& Koornneef, 2000). Drought stress leads to the over-production of reactive oxygen species (ROS) in plant cells, which in turn triggers oxidative damage and may lead to cell death (Farooq et al., 2009b; Nahar et al., 2015; Xiao et al., 2019). However, plants have evolved efficient antioxidative defense mechanisms, including the activities of antioxidant enzymes and the regulation of non-enzymatic antioxidants to reduce 
62 ROS induced oxidative damages (Posmyk et al., 2009). Plant hormones are important signaling 63 molecules that respond to environmental changes in seed germination (Yamaguchi, Kamiya \& 64 Nambara, 2007). Gibberellic acid (GA) and abscisic acid (ABA) are well-known phytohormones 65 that play crucial roles in seed germination and early seedling establishment (Shu et al., 2018; 66 Ahammed et al., 2020a). ABA, a universal abiotic stress hormone, responded positively to 67 abiotic stress, while GA acts as a plant growth regulator to promote seed germination in response 68 to stress (Shu et al., 2018). Drought stress adversely affects seed germination by disrupting the 69 dynamic balance of ABA catabolism and GA biosynthesis (Vishal \& Kumar, 2018; Ahammed et 70 al., 2020a). A previous study reported the effects of GA and salinity on physiological characteristics in maize, and their findings showed that GA could reduce proline accumulation and electrolyte leakage, alleviating the damage to plants caused by salt stress by delaying the decrease of superoxide dismutase (SOD) and peroxidase (POD) (Tuna et al., 2008). Previous studies have shown that the accumulation of ABA is closely related to the generation of ROS; specifically, when the accumulation of ABA was lower, the formation of hydrogen peroxide $\left(\mathrm{H}_{2} \mathrm{O}_{2}\right)$ reduced accordingly (Liu et al., 2010; Ye et al., 2011). Drought stress negatively

regulates metabolism due to low $\alpha$-amylase activity, leading to a significant inhibition of seed germination and seedling growth (Farooq et al., 2020). The accumulation of osmoregulatory substances, such as proline, soluble sugar, and soluble protein, which can reduce the water potential and enhance the water absorption capacity of cells, ensures normal plant metabolism and improves drought resistance (Zhang et al., 2015a; Zhang et al., 2015b).

Cotton, the world's major fiber and oil crop, is also severely affected by drought. Drought weakens seedling growth by limiting seed germination, which adversely affects cotton productivity (Farah et al., 2012; Luo et al., 2018). Therefore, it is urgent to improve the rate of cotton seed germination under drought conditions.

Melatonin (N-acetyl-5-methoxytryptamine, MT), a regulatory molecule with various biological functions, has been detected in regards to the regulation of seed germination, root development, leaf senescence, and fruit maturation in plants (Ahammed et al., 2020b; Liang et al., 2018). In addition to its physiological functions, MT can also enhance plant tolerance against multiple adverse environmental stressors (Hardeland et al., 2011; Posmyk et al., 2009; Li et al., 2018; Ahammed et al., 2020b; Li et al., 2020). Numerous studies have shown that MT plays an important role in seed germination and plant growth may be associated with MT-induced 
93 changes in physiological mechanisms (Posmyk et al., 2009; Zhao et al., 2011; Kang et al., 2010).

94 Evidence has suggested that the pre-treatment of seeds with MT could improve the effectiveness

95 of pores under drought stress (Khan et al., 2019). A previous study conducted on cucumber

96 (Cucumis sativus L.) illustrated the positive influences of MT treatment in plants suffering from

97 PEG stress including the promotion of seed germination (Zhang et al., 2012). As a broad-

98 spectrum antioxidant, MT can enhance the activity of antioxidant enzymes, such as SOD and

99 POD, by lowering ROS levels, which protects the plants from stress-induced damage (Gao et al.,

100 2018; Wang, Reiter \& Chan, 2018). Previous results have shown that the appropriate

101 concentration of MT can reduce the malondialdehyde (MDA) content in soybean seedlings,

102 alleviating the damage to the membrane system and improving resistance to abiotic stress (Wei

103 et al., 2014). Treatment with exogenous MT significantly increased the content of proline,

104 soluble sugar, and soluble protein in plants, while increasing cell fluid concentrations and

105 reducing the MDA content in plants under abiotic stress (Zhang et al., 2012; Turk et al., 2014).

106 Similarly, the exogenous application of MT has been shown to enhance the activity of $\alpha$-amylase

107 in maize seeds under chilling stress (Cao et al., 2019). MT may be a plant growth hormone that

108 is closely related to auxin (IAA) in its structural components (Arnao \& Hernandez-Ruiz, 2017).

109 Phytohormones are involved in seed germination and dormancy, and the exogenous application

110 of MT can alleviate damage to seed germination caused by environmental stress by regulating

111 the synthesis of GA and the decomposition of ABA (Zhang et al., 2014).

112 Despite the several previously proposed hypotheses suggesting that the exogenous application

113 of MT may promote seed germination under drought stress (Meng et al., 2014; Zhang et al.,

114 2012), a description of the morphological and physiological mechanisms by which MT plays a

115 role in alleviating drought stress is limited. Furthermore, little is known about the effects of MT

116 on the germination of cotton seeds and its physiology and epidermal microstructure under

117 drought stress. The objectives of our study were to (1) investigate the morphological and

118 physiological changes in cotton seeds induced by soaking the seed in MT and then treating with

119 drought stress and (2) examine the effects of soaking the seed in MT on improving the

120 germination of cotton seeds grown under drought stress.

121 MATERIALS \& METHODS

122 Reagent, plant material, and experimental conditions 
123 MT (N-acetyl-5-methoxytryptamine) was purchased from Sigma-Aldrich (St. Louis, MO, USA).

124 The experiment was conducted in the Key Laboratory of Crop Growth Regulation of Hebei 125 Agricultural University, Hebei Province, China. Seeds of the widely cultivated cotton cultivar, 126 Guoxin NO. 9 were obtained from Guoxin Agricultural Research Association of Hejian, Hebei 127 Province, China.

\section{Determination of cotton drought resistance}

129 Seeds of cotton were surface sterilized with $75 \%$ ethanol for $30 \mathrm{~min}$ and then rinsed with 130 distilled water five times. Sterilized seeds were placed in Petri dishes (each with 50 seeds and 131 five replicates) containing three layers of filter paper and $10 \mathrm{~mL}$ of the following treatment 132 solutions: 5, 10, 15, or 20\% PEG. Distilled water was used as the control. Then, seeds in Petri 133 dishes were left to germinate in an incubator at $25 \pm 1^{\circ} \mathrm{C}$ in the dark. To determine the suitable 134 concentration of PEG-6000, the seed germination rate (GR) was measured at 7 days.

\section{Determination of melatonin concentration}

136 Selected sterile seeds were soaked in different concentrations of MT solutions $(10,50,100,200$, 137 and $500 \mu \mathrm{M} \mathrm{MT}$ ) for $24 \mathrm{~h}$ at $25^{\circ} \mathrm{C}$ in a darkened incubator. For the control, the sterilized seeds were soaked in distilled water. Then, 50 seeds were transferred to Petri dishes containing three layers of filter paper moistened with $10 \mathrm{~mL}$ of PEG-6000 solution to simulate drought stress and incubated at $25^{\circ} \mathrm{C}$ in a darkened growth chamber for 7 days. The selected $10 \%$ PEG-6000 concentration was established based on the trial described above. The treatments were replicated

142 five times and each replicate contained 50 seeds. The number of germinated seeds was observed 143 daily and the GR, germination potential (GP), radical length (RL), and fresh weight (FW) were 144 determined to select the effective MT concentration for cotton seed germination under drought 145 stress.

146 The seeds were pre-soaked with $100 \mu \mathrm{M} \mathrm{MT}$ for $24 \mathrm{~h}$ at $25^{\circ} \mathrm{C}$ in a darkened incubator and then 147 allowed to germinate following the same methods as described above. Distilled water was used 148 as the control. Approximately $10 \mathrm{~g}$ of embryo and radicle were harvested from each treatment at 1492,4 , and 6 days after germination, and then stored at $-80^{\circ} \mathrm{C}$ for subsequent analyses of SOD and 150 POD activities, osmoregulation, ROS, and hormone content. Four treatments were applied as 151 follows: [1] water soaking + no stress (W); [2] water soaking + 10\% PEG-6000 (W+DS); [3] 100 $\mu \mathrm{M}$ MT soaking + no stress (MT); [4] $100 \mu \mathrm{M}$ MT soaking + 10\% PEG-6000 (MT+DS). 
153

154

155

156

157

158

159

160

161

162

163

164

165

166

167

168

169

170

171

172

173

174

175

176

177

178

179

180

181

182

183

Seed coat microstructures were observed in dry, water-soaked, and $100 \mu \mathrm{M}$ MT-soaked cotton seeds for 24 hours at $25^{\circ} \mathrm{C}$ using a scanning electron microscope (SEM).

\section{Assessment of seed germination}

The number of seeds germinated was recorded daily. The length of the radicle and hypocotyl was measured with a straightedge ruler. Seeds were considered germinated when their radicle and hypocotyl length exceeded half of the seed length.

The numbers of germinated seeds were calculated as GP and GR at 3 and 7 days after germination, respectively. GP=germinated seeds on day $3 /$ total seeds $\times 100 \%$; GR= germinated seeds on day 7 / total seeds $\times 100 \%$ (Li et al., 2007; Thabet et al., 2018). Germination index (GI) and seed vigor index (VI) were calculated as follows: GI $=\sum(\mathrm{Gi} / \mathrm{i})$, where Gi is the number of germinated seeds on day i. VI = GI $\times$ RL of germinated seeds on day 7 (Li, Yu \& Yin, 2017). In addition, the RL and FW of seeds were determined on day 7 after germination. The weight of 50 seeds was recorded using an analytical balance $(0.01 \mathrm{~g})$.

\section{Measurement of SOD and POD activities and the content of MDA and ROS}

The activities of SOD and POD were estimated using the assay kits (A001-1 and A084-3,

Nanjing Jiancheng Bioengineering Institute, Nanjing, China). Crushed frozen samples (0.3 g) were homogenized with $3 \mathrm{~mL} 50 \mathrm{mM}$ phosphate buffer (PH 7.8) containing 1\% polyvinylpyrrolidone and $0.1 \%$ mercaptoethanol $(\mathrm{w} / \mathrm{v})$, followed by centrifugation at $4{ }^{\circ} \mathrm{C}$ for 20 min at 10,000 rpm. The SOD and POD activity was determined in the supernatant using a spectrophotometer at $560 \mathrm{~nm}$ and $470 \mathrm{~nm}$ based on the manufacturer's instructions, respectively. The activity of SOD and POD was presented as protein content.

The MDA content was tested using the Malondialdehyde assay kit (Nanjing Jiancheng Bioengineering Institute, China). Cotton seed tissues were fully homogenized with $\mathrm{pH} 7.8$ phosphate buffer, after centrifugation at $6,000 \mathrm{rpm}$ for $10 \mathrm{~min}$. Two milliliters of supernatant were added to a test tube contained $3 \mathrm{~mL}$ of $5 \%$ trichloroacetic acid and $1 \mathrm{~mL}$ of $0.5 \%$ thiobarbituric acid solution. Then the mixture was boiled for $10 \mathrm{~min}$, cooled rapidly, and centrifuged at 6,000 rpm for $10 \mathrm{~min}$. The light absorption value was measured in the resulting supernatant using a spectrophotometer at $532 \mathrm{~nm}$ and $600 \mathrm{~nm}$.

The $\mathrm{H}_{2} \mathrm{O}_{2}$ and $\mathrm{O}_{2}{ }^{-}$content were tested using a Hydrogen Peroxide Assay kit and Superoxide Anion Assay kit (Nanjing Jiancheng Bioengineering Institute, Nanjing, China) following the manufacturer's protocols. 
184 Determination of soluble sugar, soluble protein, proline, and amylase contents

185 The method described by Zhang et al. (2006) was adapted for the estimation of soluble sugar 186 contents with some slight modification. Briefly, $0.3 \mathrm{~g}$ seed samples were added to $9 \mathrm{~mL}$ of

187 distilled water in a boiling water bath for $30 \mathrm{~min}$. One milliliter of the supernatant was mixed 188 with $5 \mathrm{~mL}$ of sulfuric acid-anthrone reagent, followed by boiling for $10 \mathrm{~min}$ and cooling. The 189 absorption value was measured in a spectrophotometer at $620 \mathrm{~nm}$.

190 The proline content was estimated following the method described by Subramanyam, Du \& 191 Van (2019). Briefly, approximately $0.3 \mathrm{~g}$ frozen samples were homogenized with $3 \mathrm{~mL}$ of $3 \%$ 192 aqueous sulfosalicylic acid. After centrifugation, $2 \mathrm{~mL}$ of supernatant was mixed with $2 \mathrm{~mL}$ of 193 glacial acetic acid and $2 \mathrm{~mL}$ of ninhydrin reagent, and then heated in boiling water for $30 \mathrm{~min}$. 194 After cooling, the mixture was centrifuged at 10,000 rpm for $5 \mathrm{~min}$. The light absorption values 195 were recorded at $520 \mathrm{~nm}$ using a spectrophotometer.

$196 \quad \mathrm{E}$

\section{Quantification of GA and ABA}

198 GA and ABA concentrations were determined using a previously described indirect ELISA 199 technique (He, 1993). To assay GA and ABA, $0.3 \mathrm{~g}$ frozen tissue were ground in liquid nitrogen. 200 After homogenization, $4 \mathrm{~mL}$ of $80 \%$ methanol was added to the samples. The solution was then 201 incubated for $4 \mathrm{~h}$ at $4^{\circ} \mathrm{C}$ in the dark. After centrifugation at 10,000 $\mathrm{g}$ for $20 \mathrm{~min}$, the supernatant 202 was eluted using a Sep-Pak C18 cartridge and then dried under a stream of $\mathrm{N}_{2}$. The dried samples 203 were re-dissolved with $5 \mathrm{~mL}$ of eluting buffer containing $1 \mathrm{mM} \mathrm{MgCl}, 150 \mathrm{mM} \mathrm{NaCl}$, and $10 \%$ $204(\mathrm{v} / \mathrm{v})$ methanol in $50 \mathrm{mM}$ Tris. GA and ABA were determined with an immunosorbent assay 205 according to the manufacturer's instructions, and the antibodies used in this assay were all 206 monoclonal antibodies provided by China Agricultural University.

\section{Scanning electron microscopy (SEM) analysis}

208 Parts of the testa were collected from healthy cotton seeds and observed using SEM (Hitachi 209 SU8020, Japan). The collected samples were fixed using 3\% glutaraldehyde for $12 \mathrm{~h}$ at $4^{\circ} \mathrm{C}$, and 210 then washed using 0.1 M sodium phosphate buffer. Samples were fixed with 1\% osmium for $1 \mathrm{~h}$ 211 at $4^{\circ} \mathrm{C}$ and washed twice with $0.1 \mathrm{M}$ sodium phosphate buffer. Samples were dehydrated in 212 serially diluted ethanol and critical point dried using carbon dioxide. The samples were firmly 213 mounted using double-sided adhesive tape on stubs and then sprayed with gold using a vacuum 214 plating instrument. 


\section{Statistical analysis}

216 We conducted five independent replicates for germination tests. Physiological parameters were 217 assessed using three replicates. The data were statistically analyzed using IBM SPSS Statistics

21817.0 software. A least significant difference test was determined using Duncan's test to compare 219 the mean value. All data were expressed as mean \pm standard deviation (SD). Differences were 220 considered significant at $P<0.05$. The figures were developed using the GraphPad Prism 8.0 221 software.

\section{RESULTS}

\section{Selection of PEG concentration}

224 Cotton seeds treated with different concentrations of PEG-6000 (0-20\%) were used to determine 225 the optimum concentration of PEG-6000. Fig. 1 illustrates the daily germination rate of cotton 226 seeds treated with varying concentrations of PEG-6000 within 7 days of seed germination. Fig. 2 227 shows the effects of different degrees of drought stress on the seed germination rate. The 228 increase in PEG concentration resulted in a significant delay in the germination rate of seeds. 229 Treatment with 5, 10, 15, and 20\% PEG-6000 significantly reduced the germination rate by $2303.36 \%, 20.17 \%, 37.82 \%$, and $57.14 \%$, respectively, compared with the control treatment. The 231 inhibitory effect of 5\% PEG on seed germination was weak, whereas the degree of stress of $15 \%$ 232 and 20\% PEG severely inhibited seed germination; treatment with 10\% PEG created a moderate 233 level of stress on seed germination. Therefore, in subsequent experiments, 10\% PEG was 234 selected for the drought stress treatment.

235

\section{Selection of MT concentrations for seed treatment}

237 We conducted seed germination tests to determine whether different concentrations of MT affect 238 seed germination under drought stress. In addition, we measured the radical length and fresh 239 weight of the seeds 7 days after germination. As shown in Table 1, treatment with different 240 concentrations of MT limited the negative effects of PEG-induced drought stress on germination. 241 Further analysis showed that a low concentration of MT (10-50 $\mu \mathrm{M})$ had a limited effect on GP, 242 GR, RL, and FW under drought stress conditions. Pre-soaking seeds with $100 \mu \mathrm{M}$ MT was the 243 most effective at relieving drought stress, and the results showed that GP, GR, RL, and FW 244 increased by $8.99 \%, 10.42 \%, 7.62 \%$, and $53.37 \%$ respectively, compared to the $0 \mu \mathrm{M}$ MT 245 treatment. Fewer seeds germinated when the seeds were pre-soaked in $200 \mu \mathrm{M}$ or $500 \mu \mathrm{M}$ MT. 
246 Overall, the results showed that pre-treatment with MT at an appropriate concentration could 247 promote seed germination under drought stress. Based on these results, we selected $100 \mu \mathrm{M}$ MT 248 for subsequent germination tests.

\section{Effect of MT on germination indices under drought stress conditions}

250 The seeds pre-soaked in $100 \mu \mathrm{M}$ MT showed no significant change for germination indices under 251 the well-watered condition (Table 2). MT+DS treatment resulted in a significantly higher GP $252(8.9 \%)$ and GR $(9.8 \%)$ when the seeds were germinated under drought stress conditions 253 compared with the $\mathrm{W}+\mathrm{DS}$ treatment. At 10\% PEG-6000, MT acted as a good regulator, 254 significantly improving GI and VI under drought stress. Further analysis showed that the 255 treatment of MT+DS increased GI and VI by $13.4 \%$ and $16.2 \%$, respectively, compared with 256 W+DS. Therefore, pre-treatment with MT could positively affect seed vigor.

257

258

259

260

261

262

263

264

265

266

267

268

269

270

271

272

273

274

275

276

\section{Effects of melatonin on SOD and POD activities of cotton under drought stress}

We determined the changes of SOD and POD activities at different stages of germination. Fig. 3A shows that the SOD activity of each treatment increased first and then decreased, and the highest SOD activity was found 4 days after germination. Two days after seed germination under well-watered conditions, the activity of SOD significantly increased by $3.3 \%$ in MT compared with that in W. In the seeds pre-soaked with MT, when cotton seeds were grown in normal moisture conditions for 4 and 6 days, the SOD activity increased by $2.6 \%$ and $6.3 \%$, respectively, compared to the $\mathrm{W}$ treatment, but there was no statistical difference 4 days after germination. Similarly, the activity of SOD was enhanced by the pre-treatment of seeds with MT when the seeds were germinated under drought stress conditions. Compared with the W+DS treatment, the SOD activity of the MT+DS treatment significantly increased by $5.69 \%, 2.91 \%$, and $6.03 \%$ at days 2,4 , and 6 after germination, respectively.

The results of the POD activity analysis were consistent with that of the SOD activity data (Fig. 3B). In all cases, POD activity was substantially improved by pre-soaking seeds with MT compared to seeds pre-treated with distilled water. Under well-watered conditions, POD activity was increased 1.5 times by seeds pre-soaked with MT compared with the $\mathrm{W}$ treatment at 2 days after germination. Compared with $\mathrm{W}$, the POD activity of the MT treatment improved by $38.74 \%$ and $17.9 \%$ at 4 and 6 days after germination, respectively. Soaking seeds in MT enhanced seed vigor; therefore, the POD activity of MT+DS on days 2 and 4 of germination under drought stress significantly increased by $28.28 \%$ and $36.23 \%$ respectively, compared with 
277 the W+DS treatment. Six days after seed germination, the POD activity in the MT+DS treatment

278 increased by $0.6 \%$, but this increase was not statistically significant.

279

280

Effects of melatonin pre-treatment on ROS in seeds under drought stress conditions

281 The accumulation of ROS significantly increased under drought stress. On the contrary, pre-

282 soaking seeds with MT resulted in relatively low $\mathrm{H}_{2} \mathrm{O}_{2}$ and $\mathrm{O}_{2}^{-}$content (Fig. 4). The $\mathrm{H}_{2} \mathrm{O}_{2}$

283 content in the seeds pre-soaked with MT under no stress conditions significantly decreased by

$28424.4 \%, 4.3 \%$, and $31.6 \%$, respectively at 2, 4, and 6 days, compared with $\mathrm{W}$ treatment. Under

285 drought stress conditions, the MT+DS treatment caused a decrease by $37.91 \%, 2.85 \%$, and

$28630.31 \%$ at 2,4 , and 6 days after germination, respectively compared with the W+DS treatment

287 (Fig. 4A).

288 The $\mathrm{O}_{2}^{-}$content showed an upward trend over the course of seed germination. Under normal

289 growth conditions, the $\mathrm{O}_{2}{ }^{-}$content increased in the $\mathrm{W}$ treatment. However, the increased $\mathrm{O}_{2}^{-}$was

290 alleviated by pre-soaking seeds with MT. On 2, 4, and 6 days after seed germination, the content

291 of $\mathrm{O}_{2}^{-}$in the MT treatment decreased by $43.1 \%, 0.3 \%$, and $8.1 \%$, respectively, compared with

292 W. A similar effect was observed under drought stress conditions at 2, 4, and 6 days after

293 germination; specifically, the MT+DS treatment reduced $\mathrm{O}_{2}^{-}$content by $6.16 \%, 6.81 \%$, and

$2942.82 \%$, respectively, compared to the W+DS treatment. These data indicate that MT can regulate

295 ROS during germination in the drought stress treatment (Fig. 4B).

296

297 Effects of melatonin treatment on the MDA content of cotton under drought stress

298 MDA is an important indicator reflecting the degree of membrane lipid peroxidation. The data

299 showed that drought stress induces the increase in MDA content, which was mitigated by pre-

300 soaking seeds with MT. Under drought stress, the MT+DS treatment resulted in 29.07\%,

$301 \quad 15.58 \%$, and 16.83\% lower MDA content compared with the W+DS treatment at 2, 4, and 6 days

302 after seed germination, respectively, which further suggests the effects of MT as an antioxidant

303 (Fig. 5).

304 Effect of melatonin treatment on amylase activity of seeds under drought stress

305 In our experiment, $\alpha$-amylase activity was analyzed during seed germination. The activity of $\alpha$ -

306 amylase under drought stress was decreased (Fig. 6). In contrast, seed soaking with $100 \mu \mathrm{M}$ MT

307 significantly enhanced the activities of $\alpha$-amylase. Two days after germination, the activity of $\alpha$ - 
308 amylase on the MT+DS treatment significantly increased by $14.5 \%$ compared with W+DS under

309 drought stress. MT showed higher $\alpha$-amylase activity compared with W and was increased by

$3105.9 \%$ and 3.5\% at 4 and 6 days after germination under normal conditions, respectively (Fig. 6).

311 Similarly, under drought stress conditions, the $\alpha$-amylase activity in the MT+DS treatment

312 increased by $5.2 \%$ and $5.8 \%$ on the fourth and sixth day after germination, respectively,

313 compared to the W+DS treatment. Therefore, under drought stress conditions, the $\alpha$-amylase

314 activity was effectively improved in seeds pre-soaked with $100 \mu \mathrm{M}$ MT.

315 Effects of melatonin treatment on osmoregulatory substances in seeds under drought stress

316 As shown in Fig.7A, drought stress enhanced the accumulation of proline content in seeds.

317 Under well-watered conditions, seeds pre-soaked with MT displayed a negligible effect on

318 proline content. While the MT+DS treatment significantly improved the proline content

319 compared with the W+DS treatment under drought stress. Our data showed that the proline

320 content of the MT+DS treatment significantly increased by $7.11 \%, 9.92 \%$, and 3.08\% compared

321 with the $\mathrm{W}+\mathrm{DS}$ treatment at 2, 4, and 6 days after seed germination, respectively.

322 Drought stress also increased the soluble sugar content. The soluble sugar content in seeds 323 pre-soaked with MT was higher than seeds not pre-treated with MT. Under normal conditions, 324 the soluble sugar content increased by $16.37 \%, 2.85 \%$, and $28.22 \%$ in the MT treatment at 2,4 ,

325

326

327

328

329

330

331

332

333

334

335

336

337

338 and 6 days after germination, respectively, compared with $\mathrm{W}$ treatment. Under drought stress, the soluble sugar content in the MT+DS treatment was $6.91 \%, 6.82 \%$, and $6.89 \%$ higher at 2,4 , and 6 days after germination, respectively, compared with the W+DS treatment (Fig. 7B).

The soluble protein content also responded positively to pre-soaking seeds with MT. Pretreatment with MT considerably inhibited the decrease of soluble protein content under all conditions. The soluble protein content under the MT treatment was increased by $2.16 \%, 5.03 \%$, and $7.98 \%$ at 2, 4, and 6 days after seed germination, respectively, compared to the $\mathrm{W}$ treatment. Under drought stress conditions, the soluble protein content in the MT+DS treatment increased by $6.36 \%$ and $7.95 \%$, respectively, at 2 and 4 days after seed germination compared with the $\mathrm{W}+\mathrm{DS}$ treatment. However, 6 days after germination, the effect of the MT treatment on the protein content was more effective under drought stress than under the water treatment; the protein content in the MT+DS treated cotton seeds increased by $5.22 \%$ compared with the $\mathrm{W}+\mathrm{DS}$ treatment. Consequently, we can infer that MT may accelerate the germination of cotton seeds under drought stress conditions (Fig. 7C).

Peer] reviewing PDF | (2020:03:46927:1:0:REVIEW 13 May 2020) 
339 Effects of the melatonin treatment on endogenous hormones under drought stress

340 As shown in Fig. 8A, GA continuously increased over the course of germination; however,

341 drought stress conditions negatively affected GA biosynthesis. The GA content was greater in

342 the seeds pre-soaked with MT compared with those not pre-soaked with MT under drought stress

343 conditions. The GA content increased by $10.81 \%$ in the MT+DS treatment, compared with the

$344 \mathrm{~W}+\mathrm{DS}$ treatment 2 days after germination under drought stress conditions; however, this

345 difference was not significant. Four days after germination, the GA accumulation significantly

346 increased by $16.09 \%$ in the MT+DS treatment compared with the $\mathrm{W}+\mathrm{DS}$. Thus, this treatment

347 alleviated the inhibitory effects of drought stress. At the end of the germination experiment, pre-

348 treatment with MT further promoted GA synthesis in seeds, but there was no significant

349 difference compared with the W+DS treatment. The effect of MT on drought stress during

350 germination could be via enhancing GA biosynthesis.

351 Similar to GA, the ABA content increased over the course of germination (Fig. 8B). In this

352 study, MT accelerated ABA catabolism during germination in all cases. In response to drought

353 induced by treatment with PEG-6000, ABA catabolism was limited during seed germination.

354 Additionally, the ABA content in the seeds pre-soaked with MT was lower compared to the W

355 treatment under normal conditions. Under drought stress conditions, the ABA content of the

$356 \mathrm{MT}+\mathrm{DS}$ treatment decreased by $6.22 \%, 4.2 \%$, and $6.42 \% 2,4$, and 6 days after germination,

357 respectively, compared with the $\mathrm{W}+\mathrm{DS}$. These results indicated that the mechanism by which

358 MT reduced PEG-6000 stress was by promoting the degradation of ABA.

359 Microstructural observation of testa

360 Fig. 9A shows that the seed coat stomata were rare on the surface of dry seeds. Only a small

361 number of pores were observed in seeds soaked in water (Fig. 9B). However, seeds soaked in

362 MT showed a greater number of pores (Fig. 9C). The stomata were located on the surface of the

363 epidermis between the ridges of the irregular epidermal cells, but the inner side of the stomata

364 was not surrounded by epidermis. The pores were oval and not indented on the surface of the dry

365 seeds (Fig. 9D). The periphery of the stomata was elliptic and ranged from oval to round toward

366 the center, but the inner guard cells were covered with cuticular remnants in seeds soaked in

367 water (Fig. 9E). However, soaking seeds with MT played a significant role in the opening of

368 pores in the testa. Compared with dry seeds and seeds soaked in water, the pores of seeds soaked

369 in MT were completely open and contained large guard cells (Fig. 9F).

Peer) reviewing PDF | (2020:03:46927:1:0:REVIEW 13 May 2020) 


\section{DISCUSSION}

371 Drought stress markedly impairs plant growth via different mechanisms and influences seed

372 germination and metabolism (Zhang et al., 2012). The immediate response of a plant to drought

373 stress can be determined by the inhibition of the germination rate. The damage caused by

374 drought stress to seed germination is mainly due to decrease in water intake and reduced energy

375 supply to seeds, inducing a series of changes in metabolism, including ROS accumulation,

376 antioxidant defense system, hormone signal transduction, and the production of osmotic

377 regulatory substances (Hussain, Farooq \& Lee, 2017; Shu et al., 2018; Sharma \& Zheng, 2019).

378 Numerous studies have demonstrated that drought stress significantly inhibits seed germination

379 in various plant species (Liu et al., 2018; Luo et al., 2017; Liu et al., 2016; Liu et al., 2019). Our

380 experiments showed complementary evidence that the germination rate of cotton seeds was

381 inhibited by the application of 10\% PEG-6000 (Fig. 2) because it caused a negative regulation in

382 physiological mechanisms and inhibited water absorption by seeds, suggesting that drought

383 stress affected cotton seed germination.

384 Recently, plant growth regulators have been widely used to treat seeds before sowing, and can

385 improve their resistance to abiotic stressors and promote seed germination (Liang et al., 2018;

386 Zheng et al., 2016). In the present study, seed germination inhibition caused by PEG-6000 was

387 alleviated by soaking cotton seeds with MT. Under the same drought stress treatment, seeds

388 soaked with $100 \mu \mathrm{M}$ MT showed an accelerated seed germination rate and improved radical

389 length and fresh weight compared with seeds not pre-treated with MT. In addition, 10-50 $\mu \mathrm{M}$

390 MT treatments had little effect on cotton seed germination. However, 200-500 $\mu \mathrm{M}$ MT exerted a

391 significantly negative effect on cotton seed germination (Table 1). These results demonstrated

392 that the effect of MT on cotton seeds is closely associated with its concentration and $100 \mu \mathrm{M}$ MT

393 was defined as the optimum concentration to promote cotton seed germination under drought

394 stress. These findings are in accordance with Simlat et al. (2018), who demonstrated that a low

395 concentration of exogenous MT promoted seed germination, while treatments with a high dose

396 of MT inhibited seed germination. Previous studies reported that soaking seeds in exogenous MT

$397(20$ and $100 \mu \mathrm{M})$ alleviated drought-induced germination inhibition by advancing the antioxidant

398 defense system (Ding, Liu \& Zhang, 2017). The germination test indicated that treatment with

$399100 \mu \mathrm{M}$ MT (MT+DS) increased the GP, GR, GI, and VI compared with the seeds only pre-

400 soaked with water (W+DS) under drought stress conditions (Table 2). Our findings are consistent 
401 with previous results showing that the application of exogenous MT could alleviate the inhibition 402 of PEG stress on cucumber seed germination (Zhang et al., 2014).

403 The decisive factors for seed germination are water and oxygen, which are absorbed and 404 transmitted through the seed coat (Leónie \& Maarten, 2008; Kebede, Smith \& Ray, 2014). The 405 increase in seed coat stomata promotes the intake of water and oxygen from the environment, 406 resulting in seed germination (López-Ribera \& Carlos, 2017; Wang \& Hasenstein, 2016); 407 therefore, the seed coat stomata play a vital role in seed germination and growth. The stomata 408 consist of two guard cells and subsidiary cells (Wang \& Hasenstein, 2016). Previous studies have 409 shown that the absorption rate of water is highly related to pore density (Paiva, Lemos \& 410 Oliveiva, 2006). The number of seed stomata is not necessarily species-specific and may depend 411 on environmental conditions (Wang \& Hasenstein, 2016). In the current study, few stomata were 412 observed in dry seeds, suggesting that the lack of stomata may be due to dehydration in maturing 413 seeds. The variability in the number and shapes of pores indicates that the number of stomata is 414 fluid and may have some physiological function (Wang \& Hasenstein, 2016). The results of the 415 SEM showed that pre-treatment with MT increased the number of stomata and accelerated the 416 opening of pores in testa, confirming that exogenous MT stimulated the physiological function of 417 stomata and accelerated the water absorption rate of seeds (Fig. 9). Therefore, a vital effect of the 418 MT treatment is the induction of the opening of stomata in the cotton seed coat. While a 419 quantitative analysis of stomata in testa was not carried out, our results do suggest that the 420 function of MT on the regulation mechanism of seed coat stomata requires further study.

421 Seed germination is controlled by physiological factors and ROS concentration is closely 422 correlated with water uptake by seeds during the process of germination (Moothoo et al., 2016). 423 The balance between the production and elimination of ROS is disturbed by drought stress, 424 which leads to the accumulation of ROS, including $\mathrm{O}_{2}{ }^{-}$and $\mathrm{H}_{2} \mathrm{O}_{2}$, and leads to the instability of 425 cell membranes (Kaur et al., 2015; Yang, Yao \& Zhang, 2010; Li et al., 2015; Sohag et al., 426 2020). $\mathrm{H}_{2} \mathrm{O}_{2}$ content is associated with embryo elongation during seed germination (Zhang et al., 427 2014). MT is a free radical scavenger, which may directly or indirectly eliminate ROS (Reiter et 428 al., 2007; Tan et al., 2000). Previous research has suggested that pre-treatment with MT 429 effectively inhibited the accumulation of $\mathrm{H}_{2} \mathrm{O}_{2}$ in corn seeds under stress conditions (Cao et al., 430 2019). Similarly, the application of MT reduced the accumulation of ROS and alleviated 431 membrane damage of tomato seeds and cucumber seedlings under the drought stress (Zhang et 
432 al., 2012; Liu et al., 2015). It is necessary to determine whether MT can promote the germination 433 of cotton seeds by inhibiting the accumulation of ROS. We found that seeds pre-soaked in 434 distilled water and treated with normal and dry conditions had an increase in $\mathrm{H}_{2} \mathrm{O}_{2}$ and $\mathrm{O}_{2}{ }^{-}$ 435 contents; however, the $\mathrm{O}_{2}{ }^{-}$and $\mathrm{H}_{2} \mathrm{O}_{2}$ contents were decreased in seeds pre-soaked with MT (Fig. 436 4). This may be because MT was instrumental in accelerating water absorption by the seed and 437 maintaining the balance between ROS production and clearance. Furthermore, this may have led 438 to repair of the cell membrane even under drought stress conditions. These results provided 439 evidence that exogenous MT played an important role in removing excessive ROS and 440 alleviating the membrane lipid peroxidation in cotton seeds faced with drought stress.

441 To inhibit excessive generation of ROS, plants possess a complex enzymatic defense system 442 to combat oxidative damage (Souza et al., 2014; Ahammad et al., 2020c). SOD and POD act as 443 important antioxidant enzymes that can eliminate redundant ROS from plant tissues, protecting 444 the plasma membrane from peroxidation (Xiao et al., 2019; Ramachandra et al., 2004). In plant 445 cells, SOD can rapidly convert $\mathrm{O}_{2}{ }^{-}$to form $\mathrm{H}_{2} \mathrm{O}_{2}$ and $\mathrm{O}_{2}$, while $\mathrm{H}_{2} \mathrm{O}_{2}$ can be further scavenged by 446 other antioxidant enzymes (Li et al., 2017). Many studies have shown that MT plays a vital role 447 in enhancing the activity of antioxidant enzymes and scavenging radicals in response to stress448 induced ROS damage (Zhang et al., 2014; Sharif et al., 2018; Jiang et al., 2016; Shi et al., 2015). 449 In the present study, the activity of SOD and POD were low at $2 \mathrm{~d}$ of germination, peaked at $4 \mathrm{~d}$, 450 and gradually decreased by $6 \mathrm{~d}$, indicating that the antioxidant enzyme system was active in the 451 middle of seed germination. Drought stress led to the inhibition of SOD and POD activities in 452 cotton seeds, which may be because drought stress induced the excessive generation of ROS, 453 negatively affecting antioxidant enzyme activities. We treated cotton seeds with exogenous MT 454 to enhance the tolerance of cotton seeds to drought stress. We found that seeds pre-soaked with 455 MT enhanced the activities of the SOD and POD both under normal water and drought stress 456 conditions by inhibiting the accumulation of ROS (Fig. 3). These results were in consistent with 457 a previous study that described that treatment with exogenous MT promoted SOD and POD 458 activities in grape exposed to drought conditions (Meng et al., 2014).

459 The accumulation of ROS induces lipid peroxidation and the degradation of unsaturated fatty 460 acids, producing MDA, which causes damage to the seed structure and reduces seed germination 461 (Rajjou \& Debeaujon 2008; Xiao et al., 2019). Previous studies have shown that the application 462 of exogenous MT significantly suppressed MDA accumulation under abiotic stressors in several 
463 plant species (Ahammed et al., 2019b; Hasan et al., 2019). Zhang et al. (2014) demonstrated that 464 treatment with $300 \mu \mathrm{M}$ MT, which increased the activity of antioxidant enzymes, could 465 effectively reduce the MDA content in cucumber seeds under drought stress. In the present 466 study, the MDA content gradually increased during the seed germination assay. Drought stress 467 increased the MDA content and induced lipid membrane peroxidation. However, pre-soaking 468 seeds with $100 \mu \mathrm{M}$ MT decreased the accumulation of MDA compared with seeds pre-soaked in 469 water under both normal and drought conditions (Fig. 5), suggesting that MT enhanced the 470 activity of antioxidant enzymes potentially by eliminating ROS and reducing membrane lipid 471 peroxidation in cotton seeds (Ahammed et al., 2020b).

472 Seed germination is regulated by the presence of several storage products (Waterworth et al., 473 2016; Fleming, Richards \& Walters, 2017). Amylase and osmotic regulators (e.g., proline, 474 sugars, and protein) are compatible solutes that provide necessary energy for seed 475 morphogenesis. Furthermore, amylase protects plants from stress conditions (Sadura \& 476 Janeczko, 2018) by accumulating in the form of an inactive polymer and storing in the 477 endosperm of dry seeds as storage protein, which is released and activated gradually during 478 germination; therefore, amylase plays an important role in seed germination (Swanston \& 479 Molina, 2001). Pre-treatment with exogenous MT has been revealed to increase $\alpha$-amylase 480 activity in Limonium bicolor seeds and accelerate seed germination under salt stress (Li et al., 481 2019). In the present study, under normal conditions, MT treatment was not positively correlated 482 with $\alpha$-amylase activity at $2 \mathrm{~d}$ of germination. However, the $\alpha$-amylase activity increased in 483 response to MT treatment by days 4 and 6, confirming that the effectiveness of exogenous MT 484 on amylase activity may not be apparent at the beginning of germination under normal 485 conditions. The MT+DS treatment enhanced the activity of $\alpha$-amylase at all germination time 486 points under drought stress, compared with the W+DS treatment (Fig. 6), suggesting that MT 487 may promote starch hydrolysis and seed respiration, improving seed germination and growth. 488 Proline is an important osmotic regulator and free radical scavenger that can alleviate stress 489 damage by reducing water potential (Hayat et al., 2012; Bala, 2000). We found that the proline 490 content of each treatment increased gradually throughout the process of seed germination. The 491 effect of the MT treatment was similar to that of W treatment and did not show a positive role in 492 increasing proline content. However, the MT+DS treatment performed better under drought 493 stress compared with the W+DS treatment, as evident from the increased proline content (Fig. 
494 7A). These results indicate that MT may regulate the metabolism of osmotic substances under 495 drought stress and improve drought tolerance. Pre-treatment with $100 \mu \mathrm{M}$ MT can improve the 496 drought resistance of cotton seeds, which is in agreement with a previous study that found that 497 pre-treatment with MT increases the proline content in Brassica napus exposed to Se-stress 498 (Ulhassan et al., 2019). Soluble sugar and soluble protein, which play a role in osmoregulation 499 and cell metabolism, are the main components of organelles (Wang et al., 2004; Zhang et al., 500 2015a). This study found that the content of soluble sugar and protein first increased and then 501 decreased during the process of seed germination. The contents of soluble sugar and soluble 502 protein in the MT+DS treatment were higher than in the $\mathrm{W}+\mathrm{DS}$ treatment, and the exogenous 503 application of MT may be a particularly important for the accumulation of osmotic regulators 504 under drought stress conditions (Fig. 7). These findings indicate that MT could reduce the 505 damage to plant cells caused by drought stress, reduce excessive water loss, and enhance the 506 drought resistance of plants. These findings are similar to those presented by Jiang et al. (2016). 507 Plant hormones play vital roles in the life cycle of plants, including seed germination, 508 dormancy, and response to abiotic stress (Zhou et al., 2019; Liu et al., 2016; Shu et al., 2016). It 509 is generally recognized that ABA and GA regulate seed germination antagonistically (Okamoto 510 et al., 2006); GA promotes seed germination by breaking seed dormancy, while ABA inhibits 511 seed germination (Zhou et al., 2019; Yang et al., 2014; Okamoto et al., 2006; Toh et al., 2008).

512 Pre-treatment with MT enhanced the stress resistance of plants by regulating the changes of 513 endogenous hormones (Jiang et al., 2016). Zhang et al. (2014) showed that the exogenous 514 application of MT significantly increased the GA content and accelerated ABA degradation in 515 cucumber seeds under salt stress. Our results showed that ABA content was increased, whereas 516 GA content was decreased under drought stress conditions. Compared with the W+DS treatment, 517 the MT+DS treatment increased the GA content and inhibited the production of ABA content in 518 cotton seeds grown under drought stress conditions (Fig. 8), which is indicative of the role of MT 519 on regulating the endogenous hormones in cotton under drought stress. Similarly, the pre520 treatment of cotton seeds with MT can also promote the accumulation of GA and decrease the 521 ABA content under normal water conditions; however, the effect of the MT treatment on the GA 522 content was not obvious at day 6. This may be because the effect of MT on GA biosynthesis was 523 inconspicuous at the later stage of germination in the normal water treatment. This confirmed 
524 that the application of MT can maintain the balance of GA and ABA, effectively accelerating the 525 germination of cotton seeds under drought stress.

\section{CONCLUSIONS}

527 Pre-treatment of cotton seeds with MT improves germination under drought stress conditions.

528 The results of our ultrastructure analysis of seed coats demonstrated that pre-treatment with MT

529 increased the number of stomata and promoted the opening of pores. Likely, this enhanced

530 antioxidant enzyme activities and reduced drought-induced ROS accumulation. Pre-treatment

531 with MT also increased starch metabolism and the content of osmotic substances, overall

532 enhancing the germination rate of cotton seeds. Pre-treatment with $100 \mu \mathrm{M}$ MT may promote

533 germination by regulating the hormone content; seeds pre-treated with MT reduced ABA content

534 and increased GA content. Therefore, cotton seeds soaked with MT showed a significant

535 improvement in the ability of seeds to germinate under PEG-6000 simulated drought stress. Our

536 study highlights the beneficial effects of pre-treatment with MT on the germination of cotton

537 seeds, as shown by multiple perspectives including morphological and physiological traits.

\section{ACKNOWLEDGEMENTS}

539 The authors are grateful to the anonymous reviewers for their valuable comments and

540 suggestions.

\section{REFERENCES}

542 Ahammad GJ, Li X, Yang YX, Liu CC, Zhou GZ, Wan HJ, Cheng Y. 2019a. Tomato

543 WRKY81 acts as a negative regulator for drought tolerance by modulating guard cell $\mathrm{H}_{2} \mathrm{O}_{2}-$

544 mediated stomatal closure. Environmental and Experimental Botany 171:103960 DOI

545 10.1016/j.envexpbot.2019.103960.

546 Ahammed GJ, Li Y, Cheng Y, Liu A, Chen SC, Li X. 2020a. Abscisic acid and gibberellins

547 act antagonistically to mediate epigallocatechin-3-gallate-retarded seed germination and early

548 seedling growth in tomato. Journal of Plant Growth Regulation 1-11 DOI 10.1007/s00344-020-

549 10089-1.

550 Ahammed GJ, Wu MJ, Wang YQ, Yan Y, Mao Q, Ren JJ, Ma RH, Liu A, Chen SC.

551 2020b. Melatonin alleviates iron stress by improving iron homeostasis, antioxidant defense and 552 secondary metabolism in cucumber. Scientia Horticulturae 265:109205 DOI

553 10.1016/j.scienta.2020.109205. 
554 Ahammed GJ, Wang YQ, Mao Q, Wu MJ, Yan Y, Ren JJ, Wang XJ, Liu A, Chen SC.

555 2020c. Dopamine alleviates bisphenol A-induced phytotoxicity by enhancing antioxidant and 556 detoxification potential in cucumber. Environmental Pollution 259:113957 DOI

557 10.1016/j.envpol.2020.113957.

558 Ahammed GJ, Xu W, Liu A, Chen SC. 2019b. Endogenous melatonin deficiency aggravates

559 high temperature-induced oxidative stress in Solanum lycopersicum L. Environmental and

560 Experimental Botany 161:303-311 DOI 10.1016/j.envexpbot.2018.06.006.

561 Arnao MB, Hernandez-Ruiz J. 2017. Melatonin and its relationship to plant hormones. Annals 562 of Botany 121:195-207 DOI 10.1093/aob/mcx114.

563 Bala R. 2000. Metabolic engineering for stress tolerance: installing osmoprotectant synthesis

564 pathways. Annals of Botany 4:709-716 DOI 10.1006/anbo.2000.1254.

565 Cao QJ, Li G, Cui ZG, Yang FT, Jiang XL, Diallo L, Kong F. 2019. Seed priming with

566 melatonin improves the seed germination of waxy maize under chilling stress via promoting the

567 antioxidant system and starch metabolism. Scientific Reports 9:15044 DOI 10.1038/s41598-019568 51122-y.

569 Debeaujon I, Léon-Kloosterziel KM, Koornneef M. 2000. Influence of the testa on seed 570 dormancy, germination, and longevity in Arabidopsis. Plant Physiology 122:403-14 DOI 571 10.1104/pp.122.2.403.

572 Ding F, Liu B, Zhang S. 2017. Exogenous melatonin ameliorates cold-induced damage in 573 tomato plants. Scientia Horticulturae 219:264-71 DOI 10.1016/j.scienta.2017.03.029.

574 Ehab A, Ibrahim. 2016. Seed priming to alleviate salinity stress in germinating seeds. Journal 575 of Plant Physiology 192:38-46 DOI 10.1016/j.jplph.2015.12.011.

576 Farooq M, Irfan M, Aziz T, Ahmad I, Cheema SA. 2013. Seed priming with ascorbic acid 577 improves drought resistance of wheat. Journal of Agronomy and Crop Science 199(1) DOI 578 10.1111/j.1439-037X.2012.00521.x.

579 Farooq M, Romdhane L, Sulti MK, Rehman A, Albusaidi WM, Lee DJ. 2020.

580 Morphological, physiological and biochemical aspects of osmopriming-induced drought 581 tolerance in lentil. Journal of Agronomy and Crop Science 2:176-186 DOI 10.1111/jac.12384.

582 Farooq M, Wahid A, Ito O, Lee DJ, Siddique KHM. 2009a. Advances in drought resistance 583 of rice. Critical. Reviews in Plant Sciences 28:199-217 DOI 10.1080/07352680902952173. 
584 Farooq M, Wahid A, Kobayashi N, Fujita D, Basra SMA. 2009b. Plant drought stress:

585 effects, mechanisms and management. Agronomy for Sustainable Development 29:185-212 DOI

586 10.1051/agro:2008021.

587 Farah D, Ashutosh KP, Sanjay R, Mishra A, Singh R, Sharma YK, Pramod AS, Pandey V.

588 2012. Physiological and proteomic responses of cotton (Gossypium herbaceum L.) to drought

589 stress. Plant Physiology and Biochemistry 53:6-18 DOI 10.1016/j.plaphy.2012.01.002.

590 Fleming MB, Richards CM, Walters C. 2017. Decline in RNA integrity of dry-stored soybean

591 seeds correlates with loss of germination potential. Journal of Experimental Botany 68:2219-

5922230 DOI 10.1093/jxb/erx100.

593 Gao WY, Zhang YJ, Feng Z, Bai QQ, He JJ, Wang YJ. 2018. Effects of melatonin on

594 antioxidant capacity in naked oat seedlings under drought stress. Molecules 23:1580 DOI

$59510.3390 /$ molecules23071580.

596 Hasan KH, Ahammed GJ, Sun SC, Li MQ, Yin HQ, Zhou J. 2019. Melatonin inhibits

597 cadmium translocation and enhances plant tolerance by regulating sulfur uptake and assimilation

598 in solanum lycopersicum L. Journal of Agricultural and Food Chemistry 67:10563-10576 DOI

599 10.1021/acs.jafc.9b02404.

600 Hayat S, Hayat Q, Alyemeni MN, Wani AS, Pichtel J, Ahmad A. 2012. Role of proline under 601 changing environments: a review. Plant Signaling \& Behavior 7:1456-1466 DOI

602 10.4161/psb.21949.

603 Hardeland R, Cardinali DP, Srinivasan V, Spence DW, Brown GM, Pandi-Perumal SR.

604 2011. Melatonin-A pleiotropic, orchestrating regulator molecule. Progress in Neurobiology

605 93:350-384 DOI 10.1016/j.pneurobio.2010.12.004.

606 He Z. 1993. Experimental Guide for Crop Chemical Control. Beijing Agricultural University

607 Press (in Chinese).

608 Hung B, Chen YE, Zhao YQ, Ding CB, Liao JQ, Hu C, Zhou LJ, Zhang ZW, Yuan S,

609 Yuan M. 2019. Exogenous melatonin alleviates oxidative damages and protects photosystem II

610 in maize seedlings under drought stressData_Sheet_1.ZIP. Frontiers in Plant Science 10 DOI

611 10.3389/fpls.2019.00677.

612 Hussain M, Farooq M, Lee DJ. 2017. Evaluating the role of seed priming in improving drought

613 tolerance of pigmented and non - pigmented rice. Journal of Agronomy and Crop Science 4:269-

614276 DOI $10.1111 /$ jac. 12195. 
615 Jiang CQ, Cui Q, Feng K, Xu D, Li C, Zheng Q. 2016. Melatonin improves antioxidant 616 capacity and ion homeostasis and enhances salt tolerance in maize seedlings. Acta Physiologiae.

617 Plantarum 38:82 DOI 10.1007/s11738-016-2101-2.

618 Jury WA, Vaux H. 2005. The role of science in solving the world's emerging water problems.

619 Proceedings of the National Academy of Sciences 102:15715-15720 DOI

$62010.1073 /$ pnas.0506467102.

621 Kang K, Lee K, Park S, Kim YS, Back K. 2010. Enhanced production of melatonin by ectopic 622 overexpression of human serotonin $\mathrm{N}$-acetyltransferase plays a role in cold resistance in 623 transgenic rice seedlings. Journal of Pineal Research 49:176-182 DOI 10.1111/j.1600624 079X.2010.00783.x.

625 Kaur G, Kaur S, Singh HP, Batish DR, Kohli RK, Rishi V. 2015. Biochemical adaptations in 626 Zea mays roots to short-term $\mathrm{Pb}^{2+}$ exposure: $\mathrm{ROS}$ generation and metabolism. Bull Environ 627 Conta toxico 95:246-253 DOI 10.1007/s00128-015-1564-y.

628 Kebede H, Smith JR, Ray JD. 2014. Identification of a single gene for seed coat 629 impermeability in soybean PI 594619. Theoretical and Applied Genetics 127:1991-2003 DOI $63010.1007 / \mathrm{s} 00122-014-2355-2$.

631 Khan MN, Zhang J, Luo T, Liu JH, Muhammad R, Shah F, Xu ZH, Hu LY. 2019. Seed 632 priming with melatonin coping drought stress in rapeseed by regulating reactive oxygen species 633 detoxification: Antioxidant defense, osmotic adjustment, stomatal traits and chloroplast 634 ultrastructure perseveration. Industrial Crops \& Products 140 DOI 635 10.1016/j.indcrop.2019.111597.

636 Leónie B, Maarten K. 2008. Seed dormancy and germination. American Society of Plant 637 Biologists 6:e0119 DOI 10.1199/tab.0119.

638 Liang BW, Gao TT, Zhao Q, Ma CQ. 2018. Effects of exogenous dopamine on the uptake, 639 transport, and resorption of apple ionome under moderate drought. Frontiers in Plant Science 9: 640755 DOI 10.3389/fpls.2018.00755.

641 Liang BW, Ma CQ, Zhang ZJ, Wei ZW, Gao TT, Zhao Q, Ma FW, Li C. 2018. Long-term 642 exogenous application of melatonin improves nutrient uptake fluxes in apple plants under 643 moderate drought stress. Environmental and Experimental Botany 155:650-661 DOI 644 10.1016/j.envexpbot.2018.08.016. 
645 Li C, Tan DX, Liang D, Chang C, Jia DF, Ma FW. 2015. Melatonin mediates the regulation 646 of ABA metabolism, free-radical scavenging, and stomatal behaviour in two Malus species under 647 drought stress. Journal of experimental botany 66:669-680 DOI 10.1093/jxb/eru476.

648 Li CX, Feng SI, Yun S, Jiang LN, Lu XY, Hou XL. 2007. Effects of arsenic on seed 649 germination and physiological activities of wheat seedlings. Journal of Environmental Sciences $650 \quad 19: 725-732$.

651 Li H, Chang JJ, Chen HJ, Wang ZY, Gu XR, Wei CH, Zhang Y, Ma JX, Yang JQ, Zhang 652 X. 2017. Exogenous melatonin confers salt stress tolerance to watermelon by improving 653 photosynthesis and redox homeostasis. Frontiers in Plant Science 8:295-295 DOI 654 10.3389/fpls.2017.00295.

655 Li H, Guo YL, Cui Q, Zhang ZX, Yan X, Ahammed GJ, Yang XZ, Yang JQ, Wei CH, 656 Zhang X. 2020. Alkanes (C29 and C31)-mediated intracuticular wax accumulation contributes 657 to melatonin- and ABA-induced drought tolerance in watermelon. Journal of Plant Growth 658 Regulation 1-10 DOI 10.1007/s00344-020-10099-z.

659 Li J, Zeng L, Cheng Y, Lu G, Fu G, Ma H, Liu Q, Zhang X, Zou X, Li C. 2018. Exogenous 660 melatonin alleviates damage from drought stress in Brassica napus L. (rapeseed) seedlings. Acta 661 Physiologiae Plantarum 40:1-11 DOI 10.1007/s11738-017-2601-8.

662 Li JP, Zhao C, Zhang MJ, Yuan F, Chen M. 2019. Exogenous melatonin improves seed 663 germination in Limonium bicolor under salt stress. Plant Signaling \& Behavior 14:1659705 DOI $66410.1080 / 15592324.2019 .1659705$.

665 Li XJ, Yu BJ, Yin YF. 2017. Melatonin application confers enhanced salt tolerance by 666 regulating $\mathrm{Na}^{+}$and $\mathrm{Cl}^{-}$accumulation in rice. Plant Growth Regulation 83:441-454 DOI 667 10.1007/s10725-017-0310-3.

668 Liu J, Hasanuzzaman M, Wen HL, Zhang J, Peng T, Sun HE, Zhao QZ. 2019. High

669 temperature and drought stress cause abscisic acid and reactive oxygen species accumulation and 670 suppress seed germination growth in rice. Protoplasma 256:1217-1227 DOI 10.1007/s00709671 019-01354-6.

672 Liu J, Wang W, Wang L, Sun Y. 2015. Exogenous melatonin improves seedling health index 673 and drought tolerance in tomato. Plant Growth Regulation 77:317-326 DOI 10.1007/s10725-015674 0066-6. 
675 Liu M, Wang Z, Xiao HM, Yang Y. 2018. Characterization of tadreb1 in wheat genotypes with 676 different seed germination under osmotic stress. Hereditas 155:26 DOI 10.1186/s41065-018677 0064-6.

678 Liu X, Hu P, Huang M, Tang Y, Li Y, Li L, Hou X. 2016. The NF-YC-RGL2 module 679 integrates GA and ABA signalling to regulate seed germination in Arabidopsis. Nature 680 Communications 7:12768 DOI 10.1038/ncomms12768.

681 Liu Y, Xu H, Wen XX, Liao YC. 2016. Effect of polyamine on seed germination of wheat 682 under drought stress is related to changes in hormones and carbohydrates. Journal of Integrative 683 Agriculture 15:2759-2774 DOI 10.1016/S2095-3119(16)61366-7.

684 Liu Y, Ye N, Liu R, Chen M, Zhang J. 2010. $\mathrm{H}_{2} \mathrm{O}_{2}$ mediates the regulation of ABA catabolism 685 and GA biosynthesis in Arabidopsis seed dormancy and germination. Journal of Experimental 686 Botany 61:2979-2990 DOI 10.1093/jxb/erq125.

687 López-Ribera I, Carlos MV. 2017. Use of ultrasonication to increase germination rates of 688 Arabidopsis seeds. Plant Methods 13:31 DOI 10.1186/s13007-017-0182-6.

689 Lou DJ, Wang HP, Liang G, Yu DQ. 2017. OsSAPK2 confers abscisic acid sensitivity and 690 tolerance to drought stress in rice. Frontiers in Plant Science 8:993 DOI 691 10.3389/fpls.2017.00993.

692 Luo Z, Liu H, Li WP, Zhao Q, Dai JL, Tian LW, Dong HZ. 2018. Effects of reduced nitrogen 693 rate on cotton yield and nitrogen use efficiency as mediated by application mode or plant density. 694 Field Crops Research 218:150-157 DOI 10.1016/j.fcr.2018.01.003.

695 Meng JF, Xu TF, Wang ZZ, Fang YL, Xi ZM, Zhang ZW. 2014. The ameliorative effects of 696 exogenous melatonin on grape cuttings under water-deficient stress: antioxidant metabolites, leaf 697 anatomy, and chloroplast morphology. Journal Pineal Research 57:200-212 DOI 698 10.1111/jpi.12159.

699 Moothoo-padayachie A, Varghese B, Pammenter NW, Govender P, Naidoo S. 2016.

700 Germination associated ROS production and glutathione redox capacity in two recalcitrant701 seeded species differing in seed longevity. Botany 94:1103-1114 DOI 10.1139/cjb-2016-0130. 702 Nahar K, Hasanuzaman M, Alam MM, Fujita M. 2015. Roles of exogenous glutathione in 703 antioxidant defense system and methylglyoxal detoxification during salt stress in mung bean. 704 Biologia Plantarum 59:745-756 DOI 10.1007/s10535-015-0542-x. 
705

706

707

708

709

710

711

712

713

714

715

716

717

718

719

720

721

722

723

724

725

726

727

728

729

730

731

732

733

734

735

Okamoto M, Kuwahara A, Seo M, Kushiro T, Asami T, Hirai N, Kamiya Y, Koshiba T, Nambara E. 2006. CYP707A1 and CYP707A2, which encode abscisic acid 8 '-hydroxylases, are indispensable for proper control of seed dormancy and germination in Arabidopsis. Plant Physiology 141:97-107 DOI 10.1104/pp.106.079475.

Paiva EAS, Lemos JP, Oliveira DMT, 2006. Imbibition of Swietenia macrophylla (Meliaceae) seeds: The role of stomata. Annals of Botany 98:213-217 DOI 10.1093/aob/mcl090.

Posmyk M, Bałabusta M, Wieczorek M,Sliwinska E, Janas KM. 2009. Melatonin applied to cucumber (Cucumis sativus L.) seeds improves germination during chilling stress. Journal of pineal research 46:214-223 DOI 10.1111/j.1600-079X.2008.00652.x.

Rajjou L, Debeaujon I. 2008. Seed longevity: Survival and maintenance of high germination ability of dry seeds. Comptes Rendus Biologies 331:796-805 DOI 10.1016/j.crvi.2008.07.021.

Ramachandra RA, Chaitanya KV, Jutur PP, Sumithra K. 2004. Differential antioxidative responses to water stress among five mulberry (Morus alba L.) cultivars. Environmental and Experimental Botany 52:33-42 DOI 10.1016/j.envexpbot.2004.01.002.

Reiter RJ, Tan DX, Terron MP, Flores LJ, Czarnocki Z. 2007. Melatonin and its metabolites: new findings regarding their production and their radical scavenging actions. Acta Biochimica Polonica 54:1-9 DOI 10.18388/abp.20073264.

Sadura I, Janeczko A. 2018. Physiological and molecular mechanisms of brassinosteroidinduced tolerance to high and low temperature in plants. Biologia Plantarum 62:1-16 DOI $10.1007 / \mathrm{s} 10535-018-0805-4$.

\section{Sharif R, Xie C, Zhang H, Arnao MB, Ali M, Ali Q, Muhammad I, Shalmani A, Nawaz}

MA, Chen P. 2018. Melatonin and its effects on plant systems. Molecules 23:2352 DOI 10.3390/molecules23092352.

Sharma A, Zheng BS. 2019. Melatonin Mediated Regulation of Drought Stress: Physiological and Molecular Aspects. Plants 8(7):190 DOI 10.3390/plants8070190.

\section{Shi H, Jiang C, Ye T, Tan DX, Reiter RJ, Zhang H, Liu R, Chan Z. 2015. Comparative} physiological, metabolomic, and transcriptomic analyses reveal mechanisms of improved abiotic stress resistance in bermudagrass [Cynodon dactylon (L). Pers.] by exogenous melatonin. Journal of Experimental Botany 66:681-694 DOI 10.1093/jxb/eru373.

Shu K, Liu XD, Xie Q, He ZH. 2016. Two faces of one seed: Hormonal regulation of dormancy and germination. Molecular Plant 9:34-45 DOI 10.1016/j.molp.2015.08.010. 
736 Shu K, Zhou WG, Chen F, Luo XF, Yang WY. 2018. Abscisic acid and gibberellins

737 antagonistically mediate plant development and abiotic stress responses. Frontiers in Plant

738 Science 9:416 DOI 10.3389/fpls.2018.00416.

739 Simlat M, Ptak A, Skrzypek E, Warchoł M, Morańska E, Piórkowska E. 2018. Melatonin

740 significantly influences seed germination and seedling growth of Stevia rebaudiana Bertoni. Peer

741 J 6:e5009 DOI 10.7717/peerj.5009.

742 Sohag AAM, Tahjib-Ul-Arif M, Brestic M, Afrin S, Sakil MA, Hossain MT, Hossain MA,

743 Hoddain MAF. 2020. Exogenous salicylic acid and hydrogen peroxide attenuate drought stress

744 in rice. Plant, Soil and Environment 66:7-13 DOI 10.17221/472/2019-PSE.

745 Souza TC, Magalhães PC, Castro EM, Carneiro NP, Padilha FA, Júnior CCG. 2014. ABA

746 application to maize hybrids contrasting for drought tolerance: changes in water parameters and

747 in antioxidant enzyme activity. Plant Growth Regulation 73:205-217 DOI 10.1007/s10725-013-

748 9881-9.

749 Subramanyam K, Du LG, Van DE. 2019. Sodium selenate treatment using a combination of

750 seed priming and foliar spray alleviates salinity stress in rice. Frontiers in plant science 10:116

751 DOI 10.3389/fpls.2019.00116.

752 Swanston JS, Molina-Cano JL. 2001. Beta - amylase activity and thermostability in two

753 mutants derived from the malting barley cv. Triumph. Journal of Cereal Science 33:155-161 DOI

754 10.1006/jcrs.2000.0364.

755 Tan DX, Manchester LC, Reiter RJ, Plummer BF, Limson J, Weintraub ST, QI W. 2000.

756 Melatonin directly scavenges hydrogen peroxide: a potentially new metabolic pathway of

757 melatonin biotransformation. Free Radical Biology and Medicine 29:1177-1185 DOI

758 10.1016/S0891-5849(00)00435-4.

759 Thabet SG, Moursi YS, Karam MA, Graner A, Alqudah AM. 2018. Genetic basis of drought 760 tolerance during seed germination in barley. Plos One 13 DOI 10.1371/journal.pone.0206682.

761 Toh S, Imamura A, Watanabe A, Nakabayashi K, Okamoto M, Jikumaru Y, Hanada A, 762 Aso Y, Ishiyama K, Tamura N, Kobayashi M, Yamaguchi S, Kamiya Y, Nambara E, 763 Kawakami N. 2008. High temperature induced abscisic acid biosynthesis and its role in the 764 inhibition of gibberellin action in Arabidopsis seeds. Plant Physiology 146:1368-1385 DOI 765 10.1104/pp.107.113738. Tuna AL, Kaya C, Dikilitas M, Higgs D. 2008. The combined effects 766 of gibberellic acid and salinity on some antioxidant enzyme activities, plant growth parameters 
767 and nutritional status in maize plants. Environmental and Experimental Botany 62:1-9 DOI 768 10.1016/j.envexpbot.2007.06.007.

769 Turk H, Erdal S, Genisel M, Atici O, Demir Y, Yanmis D. 2014. The regulatory effect of 770 melatonin on physiological, biochemical and molecular parameters in cold-stressed wheat 771 seedlings. Plant Growth Regulation 74:139-152 DOI 10.1007/s10725-014-9905-0.

772 Ulhassan Z, Huang Q, Gill R.A, Ali S, Mwamba TM, Ali B, Hina F, Zhou WJ. 2019.

773 Protective mechanisms of melatonin against selenium toxicity in Brassica napus: insights into 774 physiological traits, thiol biosynthesis and antioxidant machinery. BMC Plant Biology 19:507 775 DOI 10.1186/s12870-019-2110-6.

776 Vishal B, Kumar PP. 2018. Regulation of seed germination and abiotic stresses by gibberellins 777 and abscisic acid. Frontiers in Plant Science 9:838-838 DOI 10.3389/fpls.2018.00838.

778 Wang L, Hasenstein KH. 2016. Seed coat stomata of several Iris species. Flora 224:24-29 DOI 779 10.1016/j.flora.2016.07.002.

780 Wang W, Vinocur B, Shoseyov O, Altman A. 2004. Role of plant heat-shock proteins and 781 molecular chaperones in the abiotic stress response. Trends in Plant Science 9:244-252 DOI 782 10.1016/j.tplants.2004.03.006.

783 Wang Y, Reiter RJ, Chan Z. 2018. Phytomelatonin: a universal abiotic stress regulator. Journal 784 of Experimental Botany 69:963-974 DOI 10.1093/jxb/erx473.

785 Waterworth WM, Footitt S, Bray CM, Finch WE, West CE. 2016. DNA damage checkpoint 786 kinase ATM regulates germination and maintains genome stability in seeds. Proceedings of the

787 National Academy of Sciences of the United States of America 113:9647-9652 DOI

788 10.1073/pnas.1608829113.

789 Wei W, Li QT, Chu YN, Reiter RJ, Yu XM, Zhu DH, Zhang WK, Ma B, Lin Q, Zhang JS.

790 2014. Melatonin enhances plant growth and abiotic stress tolerance in soybean plants. Journal of 791 Experimental Botany 66:695-707 DOI 10.1093/jxb/eru392.

792 Xiao S, Liu LT, Wang H, Li DX, Bai ZY, Zhang YJ, Sun HC, Zhang K, Li CD. 2019.

793 Exogenous melatonin accelerates seed germination in cotton (Gossypium hirsutum L.). Plos One, 79414 DOI 10.1371/journal.pone.0216575.

795 Yamaguchi S, Kamiya Y, Nambara E. 2007. Regulation of ABA and GA levels during seed 796 development and germination in Arabidopsis. Seed Development, Dormancy and Germination 797 27:2224-247 DOI 10.1002/9780470988848.ch9. 
798 Yang RC, Yang T, Zhang HJ, Qi Y, Xing YX, Zhang N, Li R, Weeda S, Ren S, Yang B, 799 Guo YD. 2014. Hormone profiling and transcription analysis reveal a major role of ABA in 800 tomato salt tolerance. Plant Physiology and Biochemistry 77:23-34 DOI

801 10.1016/j.plaphy.2014.01.015.

802 Yang Y, Yao Y, Zhang X. 2010. Comparison of growth and physiological responses to severe 803 drought between two altitudinal hippoph aerhamnoides populations. Silva Fennica 44:603-14 804 DOI 10.14214/sf.130.

805 Yasmeen A, Basra SMA, Wahid A, Nouman W, Rehman H. 2013. Exploring the potential of 806 Moringa oleifera leaf extract (MLE) as a seed priming agent in improving wheat performance. 807 Turkish Journal of Botany 37:512-520 DOI 10.3906/bot-1205-19.

808 Ye N, Zhu G, Liu Y, Li Y, Zhang J. 2011. ABA controls $\mathrm{H}_{2} \mathrm{O}_{2}$ accumulation through the 809 induction of OsCATB in rice leaves under water stress. Plant and Cell Physiology 52:689-698 810 DOI 10.1093/pcp/pcr028.

811 Zhang HJ, Zhang N, Yang RC, Wang L, Sun QQ, Li DB, Cao YY, Weeda S, Zhao B, Ren 812 SX, Guo YD. 2014. Melatonin promotes seed germination under high salinity by regulating 813 antioxidant systems, $\mathrm{ABA}$ and $\mathrm{GA}_{4}$ interaction in cucumber (Cucumis sativus L.). Journal of 814 Pineal Research 57:269-279 DOI 10.1111/jpi.12167.

815 Zhang J, Mason AS, Wu J, Liu S, Zhang X, Luo T, Redden R, Batley J, Hu L, Yan G. 816 2015a. Identification of putative candidate genes for water stress tolerance in canola (Brassica 817 napus L.). Frontiers in Plant Science 6:1-13 DOI 10.3389/fpls.2015.01058.

818 Zhang M, Jin ZQ, Zhao J, Zhang G, Wu F. 2015b. Physiological and biochemical responses 819 to drought stress in cultivated and Tibetan wild barley. Plant Growth Regulation 75:567-574 DOI 820 10.1007/s10725-014-0022-X.

821 Zhang N, Zhao B, Zhang HJ, Weeda S, Yang C, Yang ZC, Ren SX, Guo YD. 2012.

822 Melatonin promotes water-stress tolerance, lateral root formation, and seed germination in 823 cucumber (Cucumis sativus L.). Journal of pineal research 54:15-23 DOI 10.1111/j.1600824 079X.2012.01015.x.

825 Zhang ZJ, Li HZ, Zhou WJ, Takeuchi Y, Yoneyama K. 2006. Effect of 5-aminolevulinic acid 826 on development and salt tolerance of potato (Solanum tuberosum L.) microtubers in vitro. Plant 827 Growth Regulation 49:27-34 DOI 10.1007/s10725-006-0011-9. 
828 Zhang Y, Chen BX, Xu ZJ, Shi ZW, Chen SL, Huang X, Chen JX, Wang XF. 2014.

829 Involvement of reactive oxygen species in endosperm cap weakening and embryo elongation 830 growth during lettuce seed germination. Journal of Experimental Botany 65:3189-3220 DOI $83110.093 / \mathrm{jxb} / \mathrm{eru} 167$.

832 Zhao Y, Qi LW, Wang WM, Saxena PK, Liu CZ. 2011. Melatonin improves the survival of 833 cryopreserved callus of Rhodiola crenulata. Journal of Pineal Research 50:83-88 DOI 834 10.1111/j.1600-079X.2010.00817.x.

835 Zheng MM, Tao Y, Hussain S, Jiang QW, Peng SB, Huang JL, Cui KH, Nie LX. 2016. Seed 836 priming in dry direct-seeded rice: consequences for emergence, seedling growth and associated 837 metabolic events under drought stress. Plant Growth Regulation 78:167-178 DOI 838 10.1007/s10725-015-0083-5.

839 Zhou WG, Chen F, Luo XF, Dai YJ, Yang YZ, Zheng C, Yang WY, Shu K. 2019. A matter

840 of life and death: molecular, physiological, and environmental regulation of seed longevity.

841 Plant, Cell \& Environment 2:293-302 DOI 10.1111/pce.13666.

842

843

844

845

846

Figure and table legends

Figure 1 The daily germination rate of seeds treated with different concentrations of PEG-6000.

847

848

Figure 2 Effects of drought stress on the germination rate of cotton seeds. PEG at concentrations of 0

849 (control), 5, 10, 15, and 20\% were used at the seed germination stage. Different lowercase letters indicate

850 a significant difference at the 0.05 probability level $(P<0.05)$. Error bars indicate standard errors

851 calculated for five replicates.

852

853

Figure 3 Effect of melatonin treatment on (A) superoxide dismutase (SOD) and (B) peroxidase

854

(POD) activities of cotton seeds under drought stress. Cotton seeds were pre-soaked in the solutions of 855 0 (control) and $100 \mu \mathrm{M}$ MT for $24 \mathrm{~h}$ and germination under 0 (control) and 10\% PEG stress. Different 856 lowercase letters indicate a significant difference at the 0.05 probability level $(P<0.05)$. Error bars 857 indicate standard errors calculated for three replicates.

858

859

Figure 4 Effects of pre-treatment with melatonin on $\mathrm{H}_{2} \mathrm{O}_{2}(\mathrm{~A})$ and $\mathrm{O}_{2}^{-}(\mathrm{B})$ content of cotton grown 860 under drought stress conditions. Cotton seeds were pre-soaked in the solutions of 0 (control) and 100 
$861 \mu \mathrm{M}$ MT for 24h and germination under 0 (control) and 10\% PEG stress. Different lowercase letters

862 indicate a significant difference at the 0.05 probability level $(P<0.05)$. Error bars indicate standard errors

863 calculated for three replicates.

864

865 Figure 5 Effects of melatonin treatment on MDA content of cotton under drought stress. Cotton 866 seeds were pre-soaked in the solutions of 0 (control) and $100 \mu \mathrm{M}$ MT for $24 \mathrm{~h}$ and germination under 0

867 (control) and 10\% PEG stress. Different lowercase letters indicate a significant difference at the 0.05

868 probability level $(P<0.05)$. Error bars indicate standard errors calculated for three replicates.

869

870 Figure 6 Effects of melatonin treatment on $\boldsymbol{\alpha}$-amylase activity of cotton under drought stress. Cotton 871 seeds were pre-soaked in the solutions of 0 (control) and $100 \mu \mathrm{M}$ MT for $24 \mathrm{~h}$ and germination under 0

872 (control) and 10\% PEG stress. Different lowercase letters indicate a significant difference at the 0.05

873 probability level $(P<0.05)$. Error bars indicate standard errors calculated for three replicates.

874

875 Figure 7 Effects of melatonin treatment on proline (A), soluble sugar (B), and soluble protein (C) 876 content of cotton seeds under drought stress. Cotton seeds were pre-soaked in the solutions of 0 877 (control) and $100 \mu \mathrm{M}$ MT for $24 \mathrm{~h}$ and germination under 0 (control) and 10\% PEG stress. Different 878 lowercase letters indicate a significant difference at the 0.05 probability level $(P<0.05)$. Error bars 879 indicate standard errors calculated for three replicates.

880

881

882

Figure 8. Effects of melatonin treatment on contents of gibberellin (GA) (A) and abscisic acid

883

(ABA) (B) under drought stress. Cotton seeds were pre-soaked in the solutions of 0 (control) and100

884 $\mu \mathrm{M}$ MT for $24 \mathrm{~h}$ and germination under 0 (control) and 10\% PEG stress. Different lowercase letters

885 indicate a significant difference at the 0.05 probability level $(P<0.05)$. Error bars indicate standard errors

886

887 calculated for three replicates.

888

Figure 9 Scanning electron microscopic of the testa of cotton seeds. A, D dry seed; B, E seed soaked 889

890 in water for 24 hours at $25{ }^{\circ} \mathrm{C}$; C, F seed soaked in MT solution for 24 hours at $25^{\circ} \mathrm{C}$

891

Table 1 Effects of different concentrations of melatonin on the germination and growth of seeds

$892500 \mu \mathrm{M}$ for $24 \mathrm{~h}$ and germination under $10 \%$ PEG stress. Different lowercase letters indicate a significant under drought stress. Cotton seeds were pre-soaked in MT solutions of 0 (control), 10, 50, 100, 200, and 893 difference at the 0.05 probability level $(P<0.05)$. Error bars indicate standard errors calculated for five 894 replications. 
895

896 Table 2 Effect of melatonin treatment on GP, GR, GI, and VI of cotton under drought stress. Cotton 897 seeds were pre-soaked in the solutions of 0 (control) and $100 \mu \mathrm{M}$ MT for $24 \mathrm{~h}$ and germination under 0 898 (control) and 10\% PEG stress. Different lowercase letters indicate a significant difference at the 0.05 899 probability level $(P<0.05)$. Error bars indicate standard errors calculated for five replicates. 


\section{Table $\mathbf{1}$ (on next page)}

Table 1 Effects of different concentrations of melatonin on the germination and growth of seeds under drought stress. 
1 Table 1 Effects of different concentrations of melatonin on the germination and growth of seeds

2 under drought stress. Cotton seeds were pre-soaked in MT solutions of 0 (control), 10, 50, 100, 200, and

$3500 \mu \mathrm{M}$ for $24 \mathrm{~h}$ and germination under 10\% PEG stress. Different lowercase letters indicate a significant

4 difference at the 0.05 probability level $(P<0.05)$. Error bars indicate standard errors calculated for five

5 replications.

\begin{tabular}{lllll}
\hline $\begin{array}{l}\text { Melatonin } \\
\text { concentration }\end{array}$ & GP (\%) & GR (\%) & RL (cm) & FW (g) \\
$(\boldsymbol{\mu M})$ & & & & \\
\hline 0 & $44.5 \pm 1 \mathrm{~b}$ & $72 \pm 3.65 \mathrm{c}$ & $6.3 \pm 0.16 \mathrm{~b}$ & $11.49 \pm 1.06 \mathrm{c}$ \\
10 & $43.2 \pm 2.68 \mathrm{~b}$ & $76 \pm 1.41 \mathrm{~b}$ & $6.15 \pm 0.11 \mathrm{~b}$ & $13.84 \pm 0.89 \mathrm{~b}$ \\
50 & $43.2 \pm 2.28 \mathrm{~b}$ & $73.5 \pm 1.91 \mathrm{bc}$ & $6.6 \pm 0.08 \mathrm{a}$ & $13.32 \pm 0.69 \mathrm{~b}$ \\
100 & $48.5 \pm 1.91 \mathrm{a}$ & $79.5 \pm 1.91 \mathrm{a}$ & $6.78 \pm 0.19 \mathrm{a}$ & $17.63 \pm 0.94 \mathrm{a}$ \\
200 & $33.2 \pm 2.28 \mathrm{c}$ & $63.2 \pm 1.09 \mathrm{~d}$ & $5 \pm 0.12 \mathrm{c}$ & $14.21 \pm 0.98 \mathrm{~b}$ \\
500 & $28.4 \pm 2.61 \mathrm{~d}$ & $50.5 \pm 1 \mathrm{e}$ & $2.46 \pm 0.36 \mathrm{~d}$ & $13.72 \pm 0.88 \mathrm{~b}$ \\
\hline
\end{tabular}

6 


\section{Table 2 (on next page)}

Table 2 Effect of melatonin treatment on GP, GR, Gl, and VI of cotton under drought stress. 
1 Table 2 Effect of melatonin treatment on GP, GR, GI, and VI of cotton under drought stress. Cotton

2 seeds were pre-soaked in the solutions of 0 (control) and $100 \mu \mathrm{M}$ MT for $24 \mathrm{~h}$ and germination under 0

3 (control) and 10\% PEG stress. Different lowercase letters indicate a significant difference at the 0.05

4 probability level $(P<0.05)$. Error bars indicate standard errors calculated for five replicates.

\begin{tabular}{lllll}
\hline Treatments & GP (\%) & GR (\%) & GI (\%) & VI (\%) \\
\hline W & $51.5 \pm 1 \mathrm{a}$ & $90 \pm 1 \mathrm{a}$ & $18.29 \pm 1.11 \mathrm{a}$ & $137.55 \pm 5.29 \mathrm{a}$ \\
W+DS & $44.5 \pm 1 \mathrm{c}$ & $72.25 \pm 4.03 \mathrm{c}$ & $15.37 \pm 0.64 \mathrm{c}$ & $103.44 \pm 1.83 \mathrm{c}$ \\
MT & $52.67 \pm 0.58 \mathrm{a}$ & $87.67 \pm 1.53 \mathrm{a}$ & $19.36 \pm 0.98 \mathrm{a}$ & $121.97 \pm 6.16 \mathrm{~b}$ \\
MT+DS & $48.51 \pm 0.57 \mathrm{~b}$ & $79.33 \pm 2.31 \mathrm{~b}$ & $17.44 \pm 0.61 \mathrm{~b}$ & $120.15 \pm 4.31 \mathrm{~b}$ \\
\hline
\end{tabular}

5 
Figure 1

Figure 1 The daily germination rate of seeds treated with different concentrations of PEG-6000

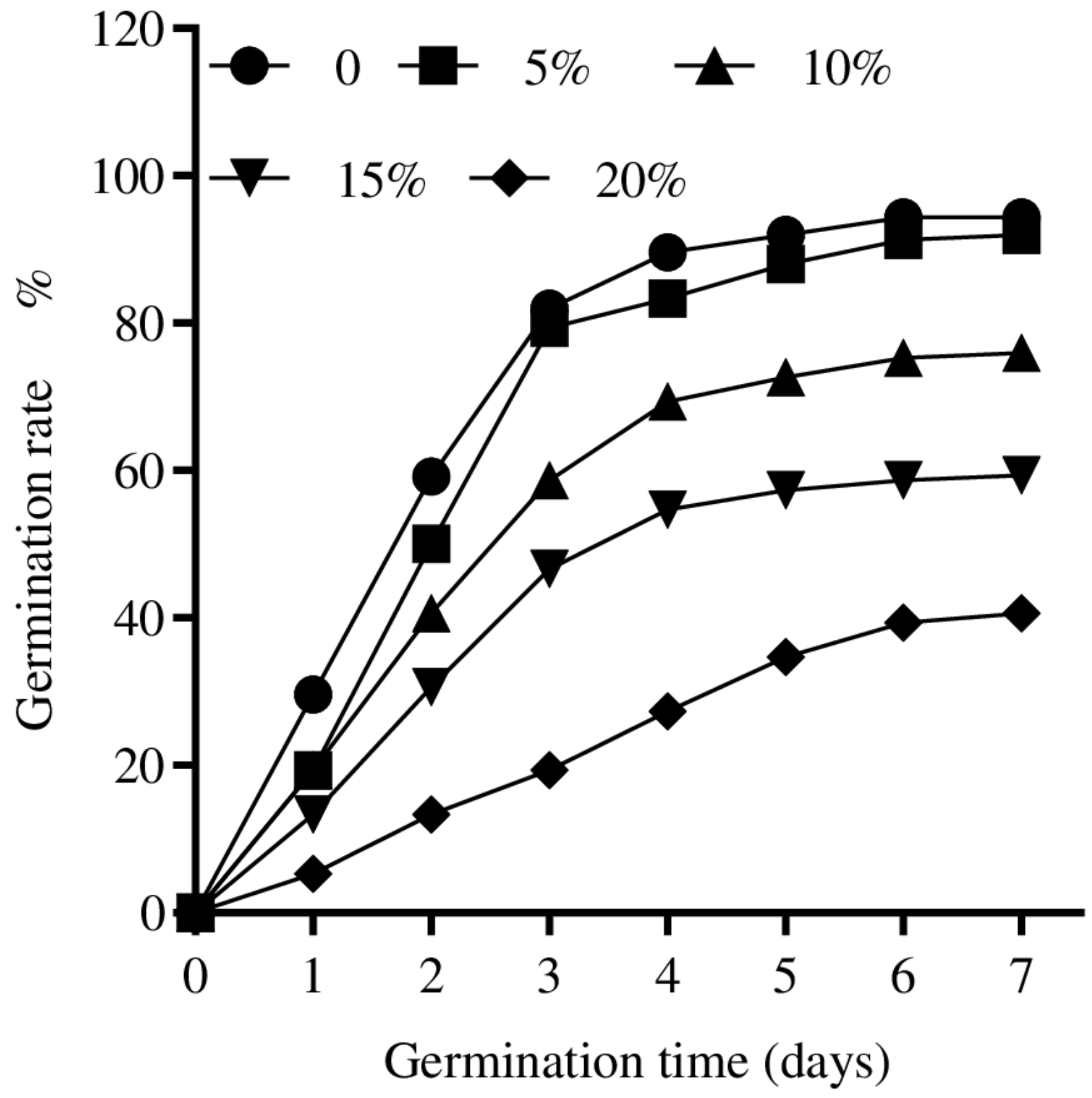


Figure 2

Figure 2 Effects of drought stress on the germination rate of cotton seeds.

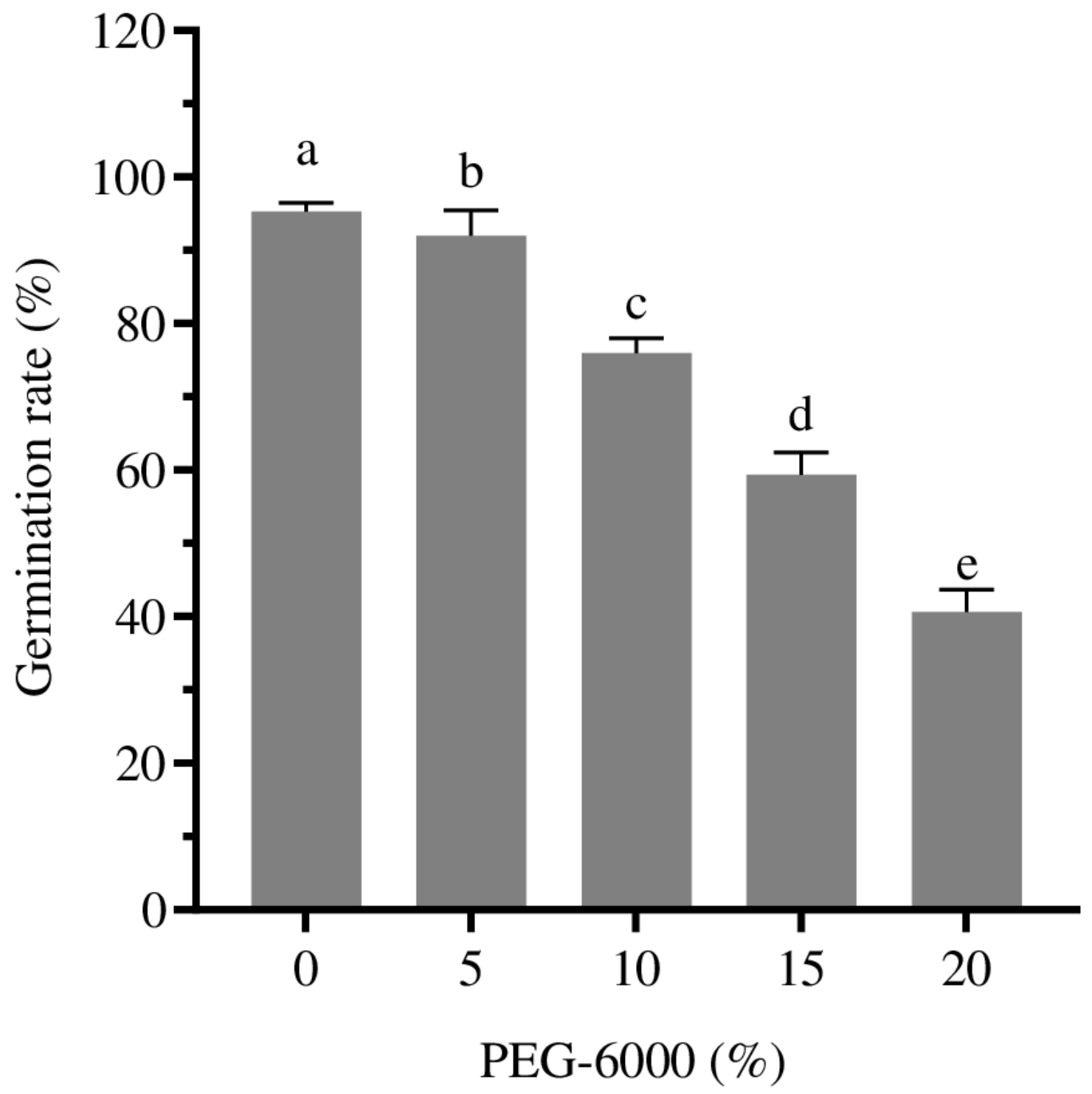


Figure 3

Figure 3 Effect of melatonin treatment on (A) superoxide dismutase (SOD) and (B) peroxidase (POD) activities of cotton seeds under drought stress.
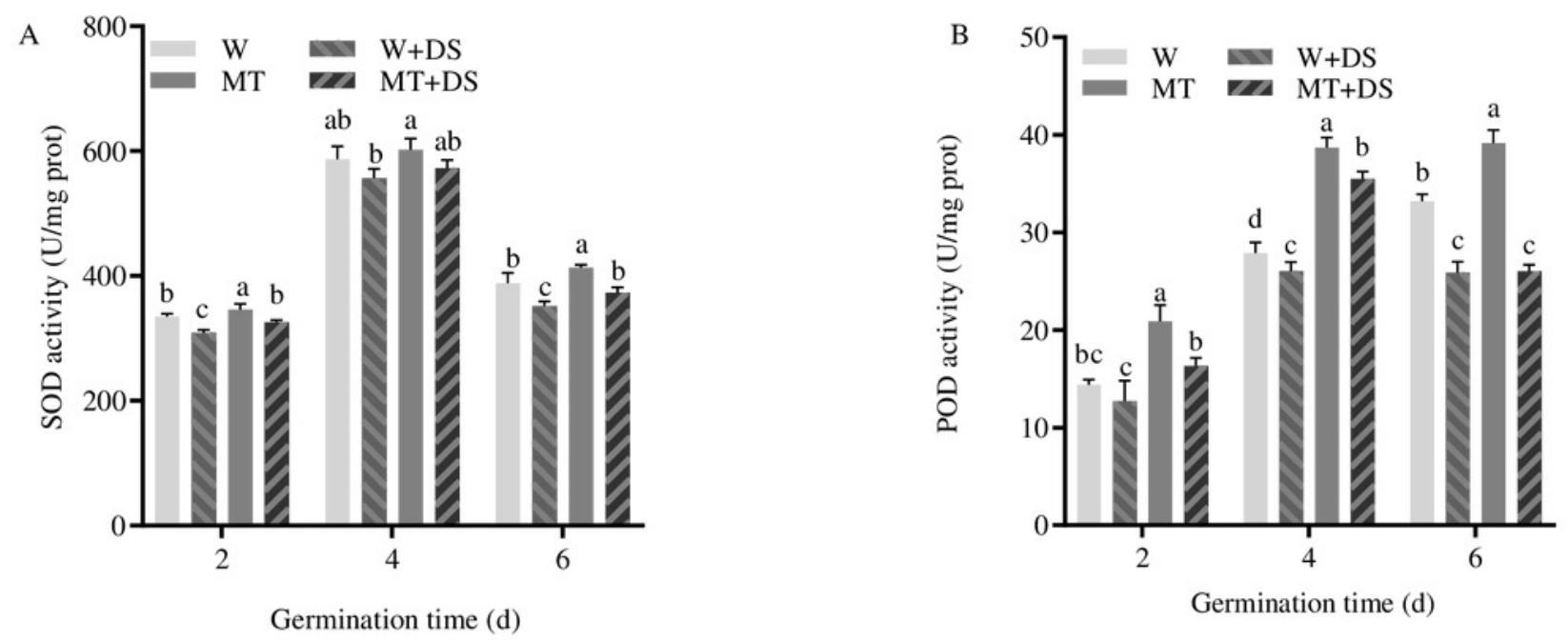
Figure 4

Figure 4 Effects of pre-treatment with melatonin on $\mathrm{H}_{2} \mathrm{O}_{2}(\mathrm{~A})$ and $\mathrm{O}_{2}{ }^{-}(\mathrm{B})$ content of cotton grown under drought stress conditions.
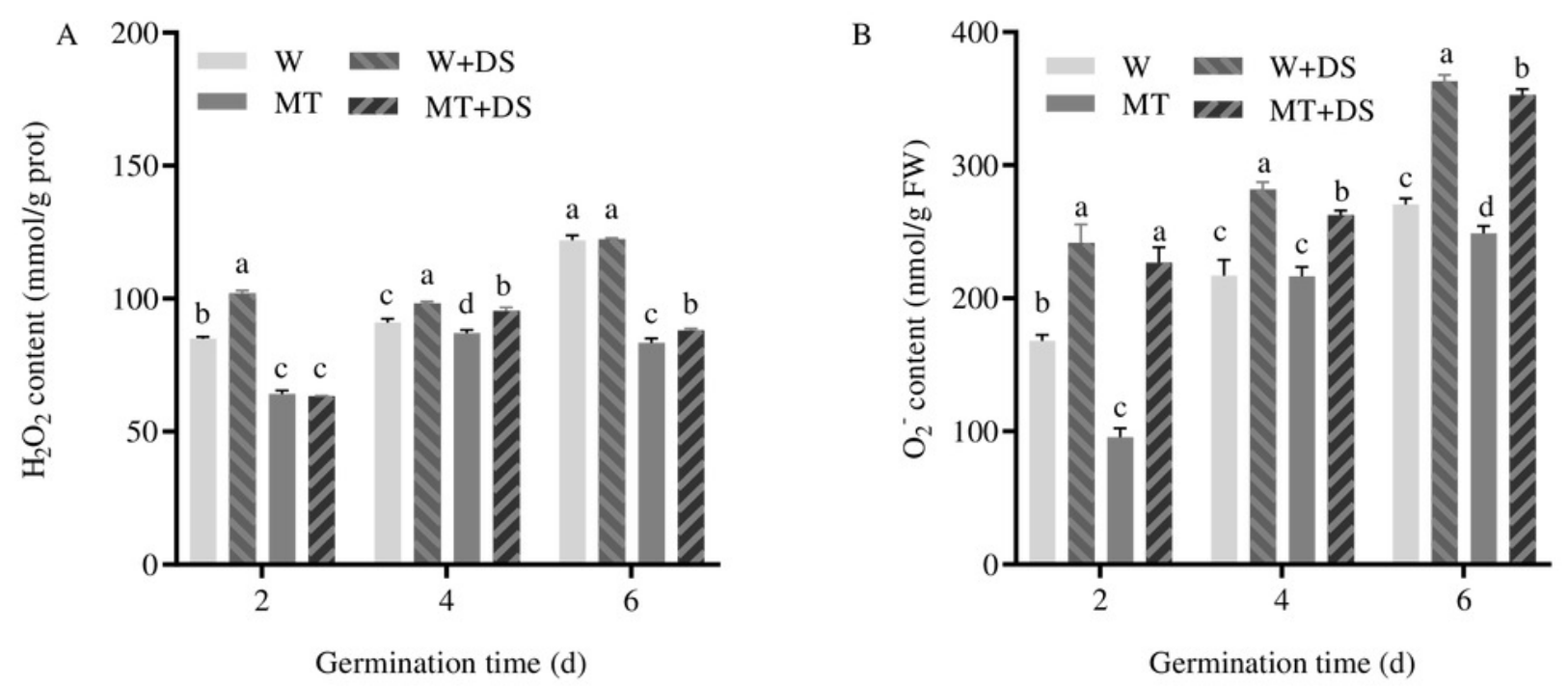
Figure 5

Figure 5 Effects of melatonin treatment on MDA content of cotton under drought stress.

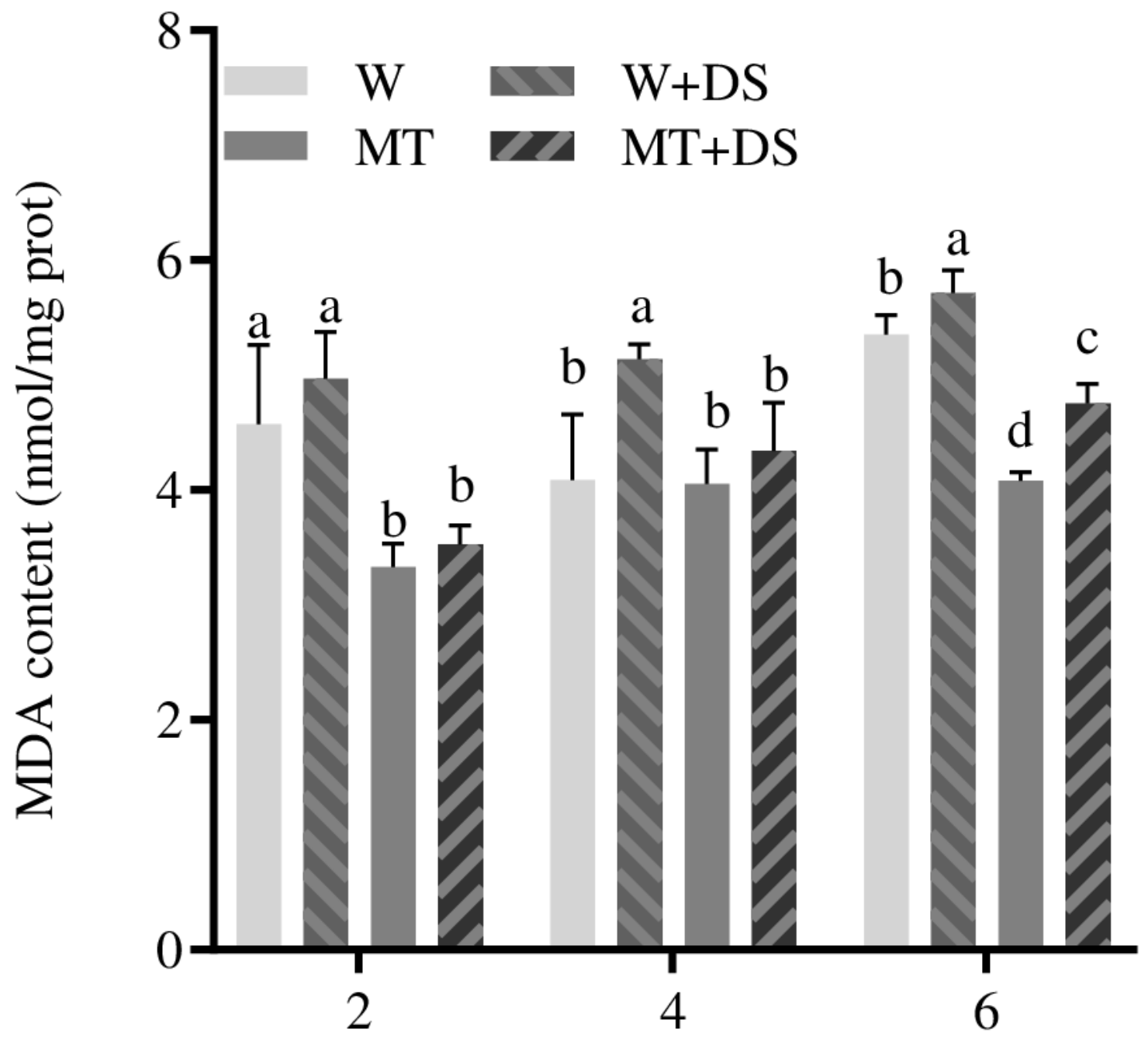

Germination time (d) 
Figure 6

Figure 6 Effects of melatonin treatment on $\alpha$-amylase activity of cotton under drought stress.

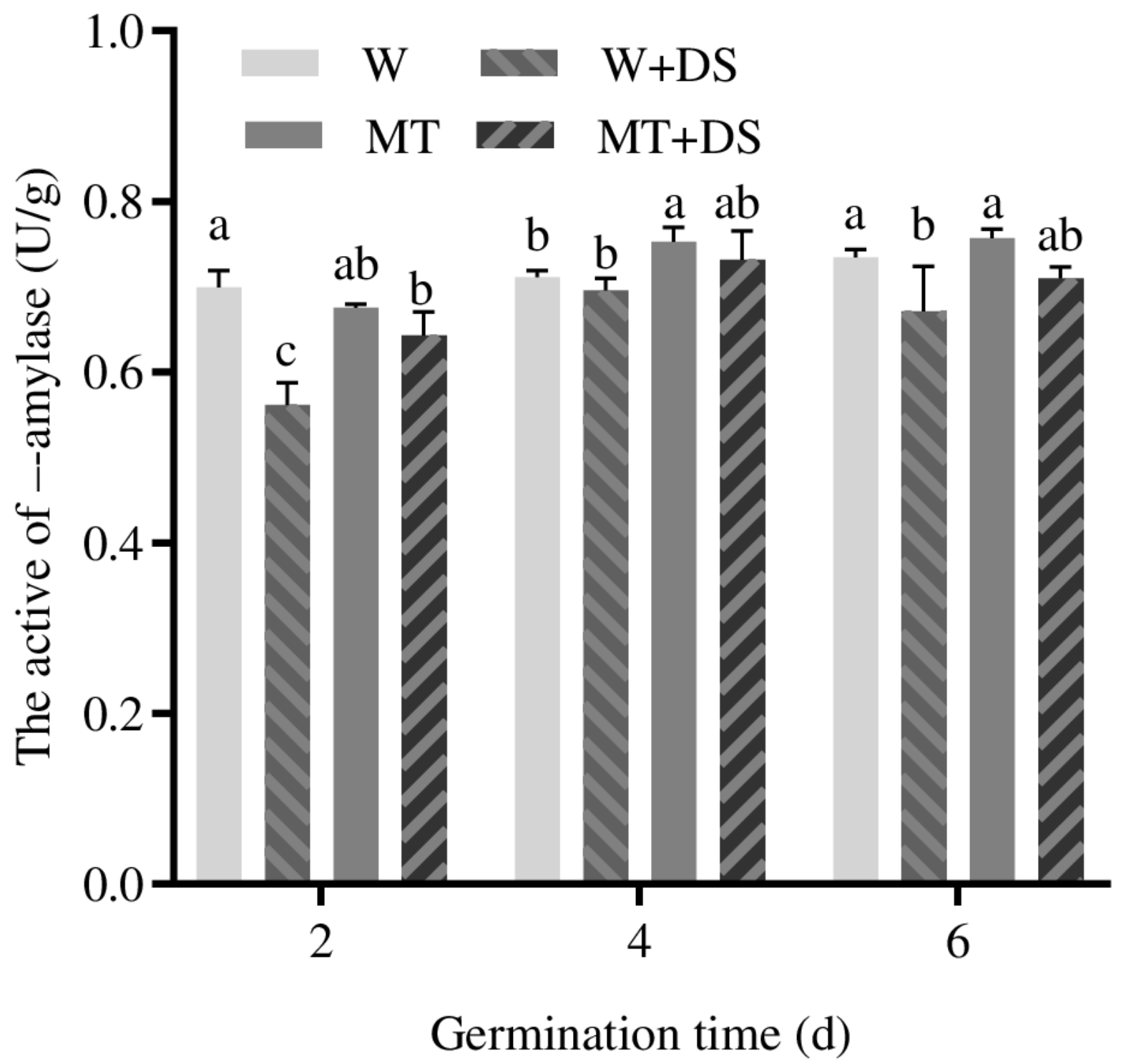


Figure 7

Figure 7 Effects of melatonin treatment on proline (A), soluble sugar (B), and soluble protein $(\mathrm{C})$ content of cotton seeds under drought stress. 

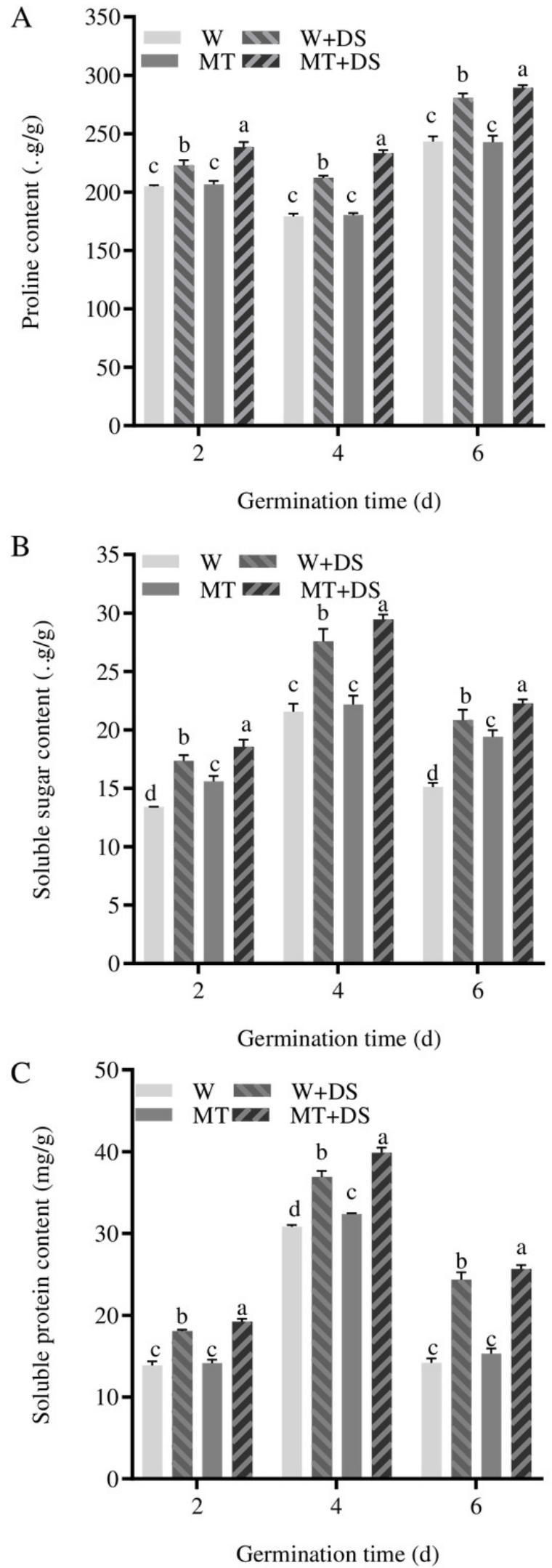
Figure 8

Figure 8. Effects of melatonin treatment on contents of gibberellin (GA) (A) and abscisic acid (ABA) (B) under drought stress.
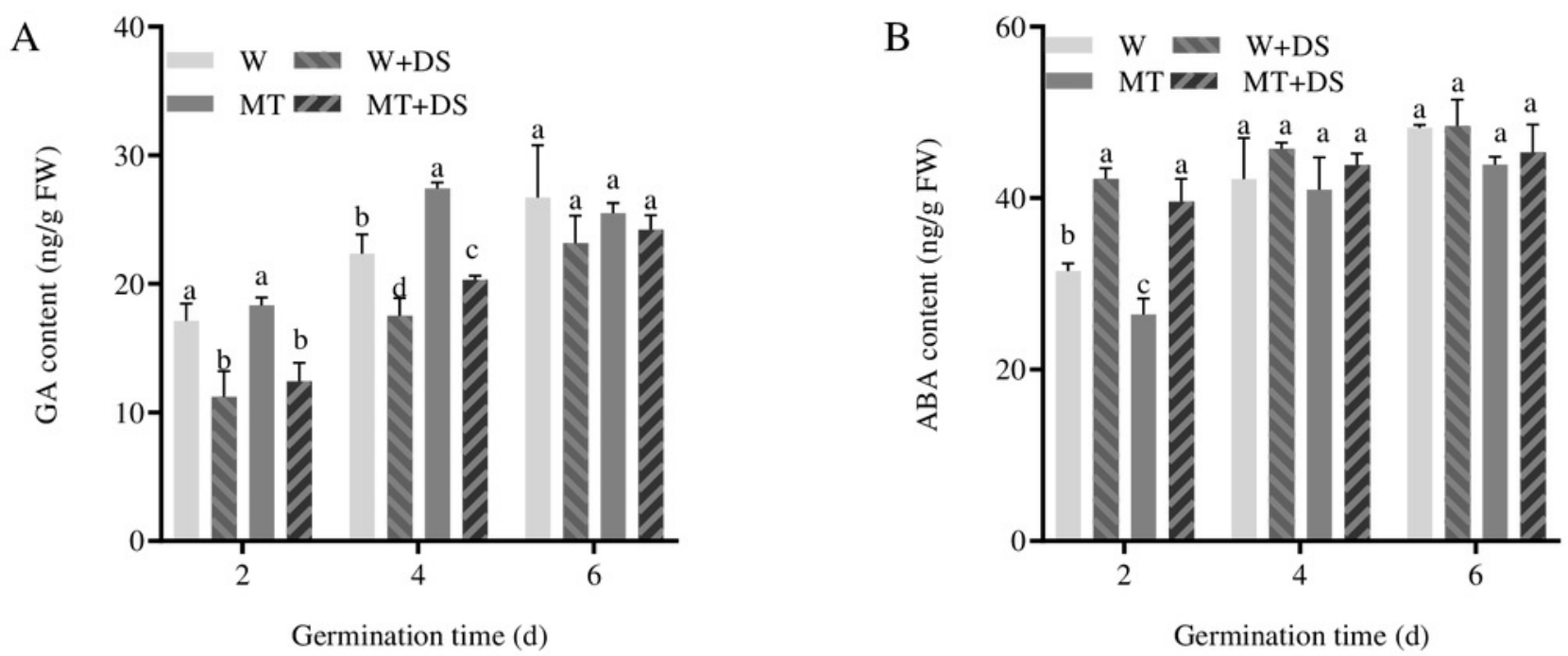
Figure 9

Figure 9 Scanning electron microscopic of the testa of cotton seeds.
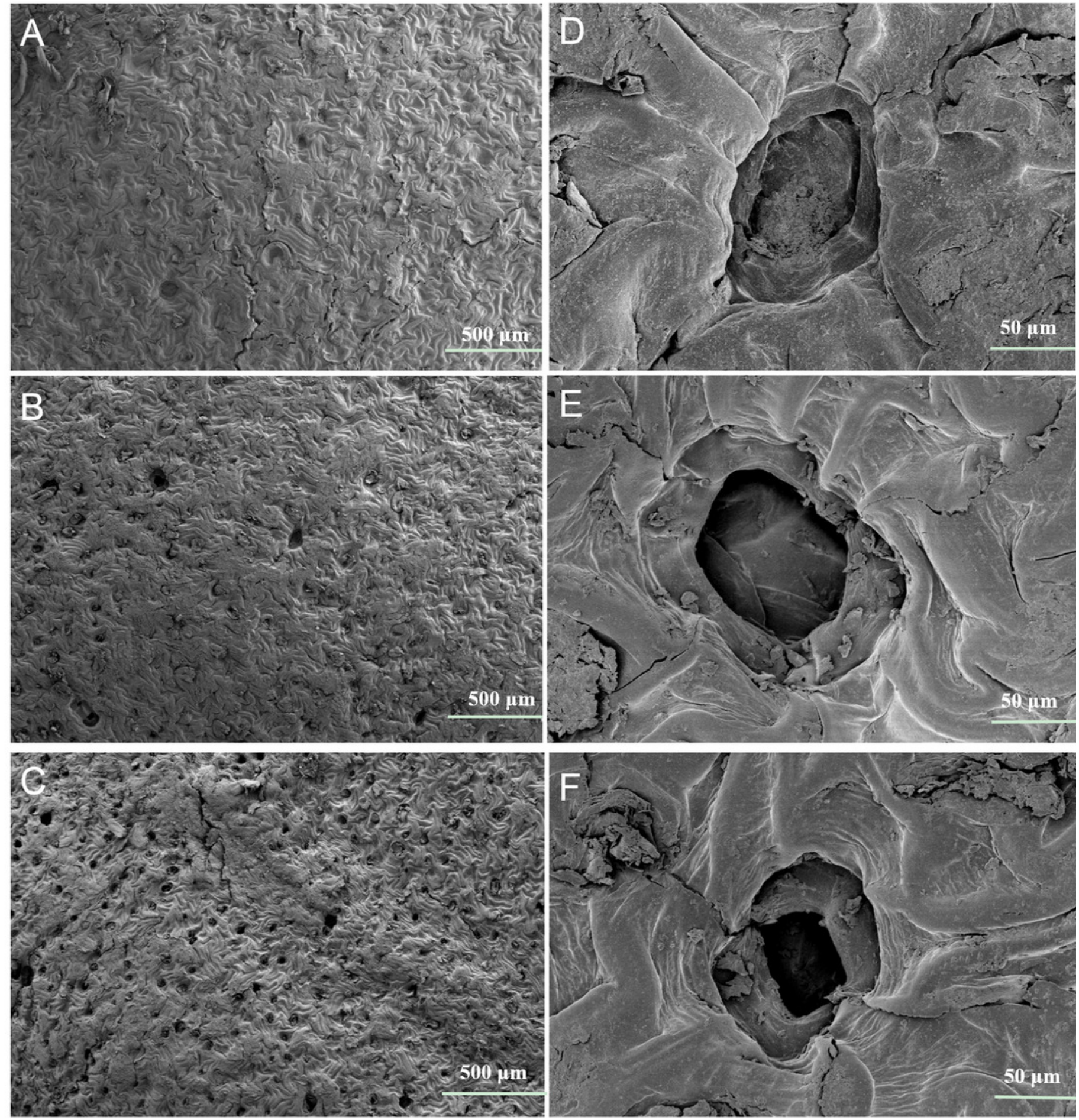